TRANSACTIONS OF THE

AMERICAN MATHEMATICAL SOCIETY

Volume 363, Number 12, December 2011, Pages 6647-6669

S 0002-9947(2011)05333-X

Article electronically published on June 15, 2011

\title{
BLOCKS WITH EQUAL HEIGHT ZERO DEGREES
}

\author{
GUNTER MALLE AND GABRIEL NAVARRO
}

\begin{abstract}
We investigate a natural class of blocks of finite groups: the blocks such that all of their height zero characters have the same degree. It is conceivable that these blocks, which are globally defined, are exactly the Broué-Puig (locally defined) nilpotent blocks and we offer some partial results in this direction. The most difficult result here is to prove that, with one family of possible exceptions, blocks with equal height zero degrees of simple groups have abelian defect groups and are in fact nilpotent.
\end{abstract}

\section{INTRODUCTION}

The celebrated nilpotent blocks of finite groups introduced by M. Broué and L. Puig in 1980 (9]) are locally defined in terms of the Alperin-Broué subpairs (1]). There is a general consensus that nilpotent blocks are the most natural blocks from the local point of view. It is not easy, however, to check if a block is nilpotent or not, and to have a global characterization of them, especially one that can be detected in the character table of the group, would be quite interesting.

Here we propose to study blocks $B$ of a finite group $G$ such that all of its height zero characters $\chi \in \operatorname{Irr}_{0}(B)$ have the same degree $d$. This property of blocks, which can easily be detected in the character table of $G$, seems to appear quite naturally in block theory and deserves some consideration. The blocks all of whose irreducible characters have the same degree were already considered by T. Okuyama and Y. Tsushima in 36.

In a nilpotent block $B$ all height zero degrees are equal, and we suspect that the converse might be true. In this paper, we are able to prove this in some cases, with quite different arguments.

If $B$ is the principal block of $G$, or if the defect group $D$ of $B$ is normal in $G$, or if $D$ is abelian (and we assume the Height Zero Conjecture), then the blocks with equal height zero character degrees are nilpotent. These results constitute Sections 3,4 , and 5 below.

The most difficult result in this paper, to which a large extent of it is devoted, is to prove that most blocks of quasi-simple groups with equal height zero degrees have abelian defect groups and satisfy Brauer's Height Zero Conjecture. By our previously mentioned result, this implies that equal height zero degrees blocks are also nilpotent. This certainly agrees with the recent work of J. An and C. Eaton in

Received by the editors September 24, 2009 and, in revised form, February 19, 2010 and February 23, 2010.

2010 Mathematics Subject Classification. Primary 20C15, 20C30, 20C33.

The first author thanks the Isaac Newton Institute for Mathematical Sciences, Cambridge, for its hospitality during the preparation of part of this work.

(C)2011 American Mathematical Society Reverts to public domain 28 years from publication 6647 
which they prove that nilpotent blocks of quasi-simple groups have abelian defect groups [2, 3]. For groups of Lie type, our argument relies on results of Lusztig, as well as on $d$-Harish-Chandra and Howlett-Lehrer theory. In the proof for alternating groups we use the fact that $p$-blocks of $\mathfrak{S}_{n}$ and certain unipotent blocks of general linear groups $\mathrm{GL}_{n}(q)$ are strongly related in order to derive in Theorem 9.1 a variant of the well-known hook formula for character degrees of symmetric groups which is particularly well-suited to block-theoretic applications.

The study of blocks of $p$-solvable groups with equal height zero degrees, which we do in the last section of the paper, leads to a variation of a classical large orbit question which does not seem easy to solve and which has interest of its own. (Some recent partial results are given in [15].) This new type of orbit problem has connections with delicate questions on the $p^{\prime}$-character degrees of finite groups.

Finally, let us mention that the blocks $B$ such that all character degrees $\chi(1)$ are $p$-powers for $\chi \in \operatorname{Irr}(B)$ give another example of blocks with equal height zero characters degrees. These blocks were proved to be nilpotent by the work of G. R. Robinson and the second author (33).

\section{EHZD BLOCKS AND NILPOTENT BLOCKS}

Suppose that $G$ is a finite group, $p$ is a prime, and $B$ is a $p$-block of $G$. In general, we use the notation in $[30$. Hence $\operatorname{Irr}(B)$ are the irreducible complex characters in $B, \operatorname{IBr}(B)$ are the irreducible Brauer characters in $B$, and $\operatorname{Irr}_{0}(B)$ are the height zero characters of $B$.

For the sake of brevity, let us say that $B$ is EHZD (equal height zero degrees) if there is an integer $d$ such that $\chi(1)=d$ for all $\chi \in \operatorname{Irr}_{0}(B)$.

Recall that a block $B$ is nilpotent if whenever $\left(Q, b_{Q}\right)$ is a $B$-subpair (that is, $b_{Q}$ is a block of $Q \mathbf{C}_{G}(Q)$ such that $\left.\left(b_{Q}\right)^{G}=B\right)$, then $\mathbf{N}_{G}\left(Q, b_{Q}\right) / \mathbf{C}_{G}(Q)$ is a $p$-group.

If $B$ is nilpotent, then we know that $\operatorname{IBr}(B)=\{\varphi\}$ by Theorem (1.2) of [9]. Also, if $\chi \in \operatorname{Irr}(B)$ has height zero, then by (3.11) on page 126 of 9 , we have that $\chi(1)=\varphi(1)$. It then follows that all irreducible height zero characters in $B$ have the same degree. Thus, as we mentioned in the introduction, nilpotent blocks are EHZD blocks. (We also notice here that in a nilpotent block all height zero characters are modularly irreducible. This condition, if not equivalent, seems also closely related to nilpotency as we shall point out in several places of this paper.)

\section{PRINCIPAL BLOCKS}

If $B$ is the principal block of $G$, then $\left(Q, b_{Q}\right)$ is $B$-subpair if and only if $b_{Q}$ is the principal block of $\mathbf{N}_{G}(Q)$ (by the Third Main Theorem). Since the principal block $b_{Q}$ is $\mathbf{N}_{G}(Q)$-invariant, we conclude that $B$ is nilpotent if and only if $\mathbf{N}_{G}(Q) / \mathbf{C}_{G}(Q)$ is a $p$-group for every $p$-subgroup $Q$ of $G$. Hence $B$ is nilpotent if and only if $G$ has a normal $p$-complement, by a classical theorem of Frobenius.

Theorem 3.1. Let $G$ be a finite group, let $p$ be a prime and let $B$ be the principal block of $G$. Then the following conditions are equivalent:

(a) All height zero $\chi \in \operatorname{Irr}(B)$ have the same degree.

(b) All height zero $\chi \in \operatorname{Irr}(B)$ are modularly irreducible.

(c) $B$ is a nilpotent block.

Proof. In Section 2 we have pointed out that (c) implies (a) and (b). Now suppose that all height zero characters in $B$ have the same degree. Hence all nonlinear 
characters in $B$ have degree divisible by $p$. Then $G$ has a normal $p$-complement by Corollary 3 of [24] and so $B$ is nilpotent.

Now, suppose that all the height zero (that is, $p^{\prime}$-degree) characters in $B$ lift an irreducible Brauer character of $G$. We are going to use a theorem of Pahlings which asserts that if $\varphi \in \operatorname{IBr}(G)$ is linear and all nonlinear characters $\chi \in \operatorname{Irr}(G)$ with decomposition number $0 \neq d_{\chi \varphi}$ have degrees divisible by $p$, then $G$ has a normal $p$-complement. (See Theorem 2 of 34.) Write $\varphi=1_{G} \in \operatorname{IBr}(G)$ for the trivial Brauer character of $G$, and suppose that $\chi$ is nonlinear with $d_{\chi \varphi} \neq 0$. If $\chi$ has $p^{\prime}$-degree, then by hypothesis, $\chi^{0}=1_{G}$ and therefore $\chi$ is linear. This is not possible. Hence, we conclude that $p$ divides $\chi(1)$. It follows that $G$ has a normal $p$-complement by Pahlings' theorem.

\section{Abelian Defect groups}

In this section we prove that EHZD blocks with abelian defect groups are exactly the nilpotent blocks (assuming Brauer's Height Zero Conjecture).

Theorem 4.1. Let $B$ be a block with an abelian defect group and assume that $\operatorname{Irr}(B)=\operatorname{Irr}_{0}(B)$. Then the following conditions are equivalent:

(a) All height zero $\chi \in \operatorname{Irr}(B)$ have the same degree.

(b) All height zero $\chi \in \operatorname{Irr}(B)$ are modularly irreducible.

(c) $B$ is a nilpotent block.

Proof. As before, we already know that (c) implies (a) and (b). By Proposition 1 of [36], we have that (a) and (b) are equivalent. Also, by Theorem 3 of [36], we have that (a) happens if and only if $B$ has inertial index one. Hence, it suffices to show that the blocks with inertial index one and abelian defect groups are nilpotent. But this is stated on page 118 (1.ex.3) of $[9$.

\section{Normal DEFECT GRoups}

In this section we prove that EHZD blocks with a normal defect group are nilpotent.

The following should be well known.

Lemma 5.1. Let $B$ be a block with defect group $D \triangleleft G$ and let $b_{D}$ be a block of $D \mathbf{C}_{G}(D)$ covered by $B$. Let $\tilde{B}$ be the Fong-Reynolds correspondent of $B$ over $b_{D}$. If $\tilde{B}$ is nilpotent, then $B$ is nilpotent.

Proof. Let $T$ be the stabilizer of $b_{D}$ in $G$. Now $\left(b_{D}\right)^{T}=\tilde{B}$, and $\left(D, b_{D}\right)$ is a $\tilde{B}$-subpair. Since $\tilde{B}$ is nilpotent, we have that $T / \mathbf{C}_{G}(D)$ is a $p$-group. Since $T / D \mathbf{C}_{G}(D)$ has order not divisible by $p$, we conclude that $T=D \mathbf{C}_{G}(D)$ and $\tilde{B}=b_{D}$. Now, suppose that $\left(Q, b_{Q}\right)$ is a $B$-subpair. We want to show that $\mathbf{N}_{G}\left(Q, b_{Q}\right) / \mathbf{C}_{G}(Q)$ is a $p$-group. For this we may replace $\left(Q, b_{Q}\right)$ by any $G$ conjugate. Since $\left(b_{Q}\right)^{G}=B$, we have that $Q \subseteq D$ (Theorem (4.14) of [30]). Now let $e=\left(b_{Q}\right)^{D \mathbf{C}_{G}(Q)}$. We have that $e^{G}=B$ by the transitivity of induction. Now, if $f$ is a block of $D \mathbf{C}_{G}(D)$ covered by $e$, we have that $e=f^{D \mathbf{C}_{G}(Q)}$ by Corollary (9.21) of 30. Hence $e^{G}=B$ and $f^{x}=b_{D}$ for some $x \in G$. Now, replacing $\left(Q, b_{Q}\right)$ by $\left(Q^{x},\left(b_{Q}\right)^{x}\right)$, we may assume that $\left(b_{D}\right)^{D \mathbf{C}_{G}(Q)}=e$. Now, suppose that 
$y$ stabilizes $\left(Q, b_{Q}\right)$. Then $y$ stabilizes $\left(b_{Q}\right)^{D \mathbf{C}_{G}(Q)}=e$. Hence $e$ covers $\left(b_{D}\right)^{y}$, and therefore $\left(b_{D}\right)^{y z}=b_{D}$ for some $z \in \mathbf{C}_{G}(Q)$. Since $T=D \mathbf{C}_{G}(D)$, we see that $y z \in D \mathbf{C}_{G}(D)$ and therefore $y \in D \mathbf{C}_{G}(Q)$. Thus $\mathbf{N}_{G}\left(Q, b_{Q}\right) / \mathbf{C}_{G}(Q)$ is a $p$-group and $B$ is nilpotent.

Theorem 5.2. Suppose that $B$ has defect group $D \triangleleft G$. Then the following conditions are equivalent:

(a) All height zero $\chi \in \operatorname{Irr}(B)$ have the same degree.

(b) All height zero $\chi \in \operatorname{Irr}(B)$ are modularly irreducible.

(c) B is a nilpotent block.

Proof. We already know that (c) implies (a) and (b). We prove by induction on $|G|$ that (a) implies (c). In an analogous way, we could prove that (b) implies (c). Let $b_{D}$ be a block of $D \mathbf{C}_{G}(D)$ inducing $B$ with defect group $D$. Let $T$ be the stabilizer in $G$ of $b_{D}$, and let $\tilde{B}$ be the Fong-Reynolds correspondent of $B$ over $b_{D}$. If all height zero $\chi \in \operatorname{Irr}(B)$ have the same degree, then the same happens in $\tilde{B}$ by the Fong-Reynolds correspondence [30, Theorem (9.14)]. By Lemma (5.1), we may assume that $T=G$. Now by Reynolds Theorems 6 and 7 of [37, there exists a group $M$ with normal Sylow $p$-subgroup $D$ such that $D \mathbf{C}_{M}(D)=D \times Z$, where $Z$ is central, and $M / D \mathbf{C}_{M}(D) \cong G / D \mathbf{C}_{G}(D)$. Also $M$ has a block $B_{1}$, with defect group $D$, having all height zero irreducible characters of the same degree and such that $\operatorname{Irr}\left(B_{1}\right)=\operatorname{Irr}(M \mid \lambda)$ for some irreducible $\lambda \in \operatorname{Irr}(Z)$. (We use $\operatorname{Irr}(M \mid \lambda$ ) to denote the irreducible characters of $M$ lying over $\lambda$.) Since $D$ is normal in $M$, the height zero characters of $B_{1}$ are exactly those irreducible characters of $B_{1}$ of $p^{\prime}$-degree. Hence, these are exactly the irreducible characters of $B_{1}$ having $D^{\prime}$ in their kernel. Now, by Theorem (9.9.b) of [30, we have that $B_{1}$ contains a block $\bar{B}_{1}$ of $M / D^{\prime}$ with defect group $D / D^{\prime}$. This block $\bar{B}_{1}$ has all irreducible characters of the same degree. By Theorem 3 of [36], we conclude that $\bar{B}_{1}$ has inertial index one. Thus

$$
\left(D / D^{\prime}\right) \mathbf{C}_{M / D^{\prime}}\left(D / D^{\prime}\right)=M / D^{\prime} .
$$

Using that a $p^{\prime}$-group acts trivially on $D$ if and only if it acts trivially on $D / D^{\prime}$, we easily conclude that $M=D \mathbf{C}_{M}(D)$ and therefore $G=D \mathbf{C}_{G}(D)$. In this case, the block is nilpotent. (See (1.ex. 1) on page 118 of 9 .)

\section{QUASI-SIMPLE GROUPS}

From now until the last section of the paper, we are devoted to proving the following result.

Theorem 6.1. Let $S$ be a finite nonabelian simple group, $G$ a quasi-simple group with $G / Z(G) \cong S$, and $p$ a prime. Assume that $B$ is a p-block of $G$ such that all characters in $\operatorname{Irr}_{0}(B)$ have the same degree. Then the defect group of $B$ is abelian and thus $B$ is nilpotent, unless possibly one of the following holds:

(1) $B$ is a faithful block for the 2-fold covering group $2 . \mathfrak{A}_{n}$ of the alternating group $\mathfrak{A}_{n}(n \geq 14)$ (a so-called spin-block), or

(2) $B$ is a quasi-isolated block for an exceptional group of Lie type and $p$ is a bad prime. 
Theorem 4.1 shows that in order to check Theorem 6.1 it suffices to prove the following for any block $B$ of $G$ all of whose characters $\operatorname{in}_{\operatorname{Irr}_{0}}(B)$ have the same degree:

(1) the defect group of $B$ is abelian, and

(2) $B$ satisfies the Height Zero Conjecture.

In order to do this, we invoke the classification of finite simple groups as well as the Deligne-Lusztig theory of characters of finite reductive groups and the fundamental results of Bonnafé-Rouquier and Cabanes-Enguehard on blocks. This will be given in several steps in the subsequent sections. The proof in the case of alternating groups will lead to a relative hook formula for the character degrees in $p$-blocks of the symmetric group.

\section{UNIPOTENT BLOCKS}

In this section we consider the unipotent blocks of finite groups of Lie type. We introduce the following standard setup: Any nonexceptional Schur covering group of a finite simple group of Lie type can be obtained as $G:=\mathbf{G}^{F}$, where $\mathbf{G}$ is a simple algebraic group of simply connected type over the algebraic closure of a finite field, and $F: \mathbf{G} \rightarrow \mathbf{G}$ a Frobenius map with finite group of fixed points $\mathbf{G}^{F}$, with the sole exception of the Tits group ${ }^{2} F_{4}(2)^{\prime}$, which will be treated later in Proposition 9.4. Let $\mathbf{G}^{*}$ denote a group in duality with $\mathbf{G}$ and with corresponding Frobenius map $F^{*}: \mathbf{G}^{*} \rightarrow \mathbf{G}^{*}$ and fixed points $G^{*}:=\mathbf{G}^{* F^{*}}$. Let $r$ denote the defining characteristic of $G$ and $q$ the absolute value of all eigenvalues of Frobenius on the character lattice of an $F$-stable torus of $G$, a half-integral power of $r$. We will then also write $G=G(q)$ in order to indicate the corresponding value of $q$.

By the fundamental work of Lusztig, the irreducible characters of $G$ can be partitioned into so-called Lusztig series

$$
\operatorname{Irr}(G)=\coprod_{(s)} \mathcal{E}(G, s)
$$

indexed by conjugacy classes of semisimple elements $s$ in $G^{*}$. The characters in the Lusztig series $\mathcal{E}(G):=\mathcal{E}(G, 1)$ corresponding to the trivial element in $G^{*}$ are the so-called unipotent characters. These can be viewed as being the building blocks of the ordinary character theory of finite groups of Lie type. Again by results of Lusztig, the unipotent characters can be parametrized by a set depending only on the type of $G$, that is, on the Weyl group of $\mathbf{G}$ together with the action of $F$ on it, not on $q$ or $r$. Moreover, their degrees are given by the value at $q$ of polynomials in one indeterminate of the form

$$
\frac{1}{n} x^{a} \prod_{i=1}^{m} \Phi_{i}(x)^{a_{i}},
$$

where $n$ is either a power of 2 or a divisor of 120 , and $\Phi_{i}(x)$ denotes the $i$ th cyclotomic polynomial over $\mathbb{Q}$ (see for example Chapter 13 of [11]). We write $\operatorname{Deg}(\gamma) \in \mathbb{Q}[x]$ for the degree polynomial of a unipotent character $\gamma \in \mathcal{E}(G)$.

7.1. Specializations of degree polynomials. We start by investigating specializations of degree polynomials of unipotent characters. We first discuss the question when two different degree polynomials $f_{1}, f_{2}$ can lead to the same character degree $f_{1}(q)=f_{2}(q)$. 
Lemma 7.1. Let $n_{1}, n_{2}, m \in \mathbb{N}, a_{i} \in \mathbb{Z}$ with $a_{m} \neq 0, q>1$ a prime power, and assume that

$$
n_{1}=n_{2} \prod_{i=1}^{m} \Phi_{i}(q)^{a_{i}}
$$

(a) If both $n_{1}, n_{2}$ are powers of 2 , then $(m, q)=(6,2)$ and $\left(a_{3}, \ldots, a_{6}\right)=$ $\left(0,0,0,-a_{2}\right)$, or $(m, q)=(2,3)$, or $m=1, q=2^{f}+1$.

(b) If both $n_{1}, n_{2}$ are divisors of 120 , then either $m=4$ and $q \in\{2,3\}$, or $m=2$ and $q \in\{2,3,4,5,7,9,11\}$, or $m=1,(q-1) \mid 120$.

Proof. First assume that there is a Zsigmondy primitive prime divisor $p_{m}$ of $\Phi_{m}(q)$, that is, $p_{m}$ divides $\Phi_{m}(q)$, but it does not divide $\Phi_{i}(q)$ for $i<m$. This is the case unless $m=1$, or $m=2$ and $q+1$ is a power of 2 , or $(m, q)=(6,2)$. Clearly, $p_{m} \neq 2,3$ if $m \neq 1,2$, and $p_{m} \neq 5$ if $m \neq 1,2,4$. Comparing prime factorizations on both sides we see that $m \in\{1,2,4\}$ if $n_{1} n_{2}$ is divisible by 5 , and $m \in\{1,2\}$ if not.

Further assume that $m=4$, so $5 \mid\left(q^{2}+1\right)$. As any two of $q-1, q+1, q^{2}+1$ have gcd at most 2 , their only prime divisors can be 2,3 and 5 , and we must have $q^{2}+1 \leq 240$. It is easy to check that this only happens for $q \in\{2,3\}$. Next assume that $m=2, q+1$ is not a power of 2 , and 5 is the largest Zsigmondy prime for $q+1$. As before, $q+1 \leq 240$, and $q-1, q+1$ are only divisible by 2,3 and 5 . We arrive at $q \in\{4,9\}$. Similarly, if $m=2$ and 3 is the only Zsigmondy prime for $q+1$, then $q \in\{2,5,11\}$.

Thus we may assume that there is no Zsigmondy prime for $\Phi_{m}(q)$, that is, $m=1$, or $m=2$ and $q+1$ is a power of 2 , or $(m, q)=(6,2)$. In the latter case, using Zsigmondy primes for $\Phi_{3}(q), \Phi_{4}(q), \Phi_{5}(q)$ we see that $a_{3}=a_{4}=a_{5}=0$. Moreover, as $\Phi_{2}(2)=3=\Phi_{6}(2)$, these two factors must occur with opposite exponent. If $m=2$, then both $q-1, q+1$ have to be powers of 2 , whence $q=3$, or both have to divide 240. The latter implies that $q \in\{3,7\}$. Finally, when $m=1$, then $q-1$ is a power of 2 or a divisor of 120 . This proves the claim.

Proposition 7.2. Let $f_{1}, f_{2} \in \mathbb{Q}[x]$ be the degree polynomials of two unipotent characters of an exceptional group of Lie type $G=G(q)$. If $f_{1}(q)=f_{2}(q)$ for some prime power $q>1$, respectively square root of some odd power of a prime for the Suzuki or Ree groups, then $f_{1}=f_{2}$, or

$$
G \in\left\{G_{2}(2),{ }^{2} B_{2}(2),{ }^{2} F_{4}(2),{ }^{2} G_{2}(3)\right\} .
$$

Proof. Write $f_{j}=\frac{1}{n_{j}} x^{a_{j}} \prod_{i=1}^{m_{j}} \Phi_{i}(x)^{a_{i, j}}$ for $j=1,2$. According to [11, Chap. 13], $n_{j} \mid 120$ for $G=E_{8}$, and $n_{j} \mid 24$ otherwise. Now $f_{1}(q)=f_{2}(q)$ implies that $q^{a_{1}-a_{2}} \prod_{i=1}^{m_{j}} \Phi_{i}(q)^{a_{i, 1}-a_{i, 2}} \in \mathbb{Q}$ has numerator and denominator a divisor of 120 . Since the second factor is coprime to $q$, this holds in fact for both factors. Then Lemma 7.1(b) shows that $q \leq 121$ for $G=E_{8}$, and $q \leq 25$ for the other types. For these finitely many values of $q$ and finitely many types, the assertion can be checked from the tables of degree polynomials. In fact, the additional restrictions in Lemma 7.1(b) allow us to restrict the number of necessary computations even further.

Note that none of the exceptions $G_{2}(2),{ }^{2} B_{2}(2),{ }^{2} G_{2}(3),{ }^{2} F_{4}(2)$ is a perfect group. Unfortunately, there are infinitely many exceptions to the conclusion of the previous proposition in the case of classical groups, so we will choose a different approach for those. 
7.2. $e$-symbols and degrees. We need to give a brief recall of the notion of $e$ symbols and associated degree; see [27.

Let $e \geq 1$ be an integer. An e-symbol is a sequence $S=\left(S_{1}, \ldots, S_{e}\right)$ of $e$ strictly increasing sequences $S_{i}=\left(s_{i 1}<\ldots<s_{i m}\right)$ of nonnegative integers of equal length $m$. The rank of an $e$-symbol $S$ is defined as

$$
\operatorname{rk}(S):=\sum_{s \in S} s-e\left(\begin{array}{c}
m \\
2
\end{array}\right) \text {. }
$$

We define an equivalence relation on $e$-symbols as the reflexive, symmetric and transitive closure of the relation $\sim$ given by

$$
\left(S_{1}, \ldots, S_{e}\right) \sim\left(S_{1}^{\prime}, \ldots, S_{e}^{\prime}\right) \Longleftrightarrow S_{i}^{\prime}=\left(0, s_{i 1}+1, \ldots, s_{i m}+1\right) .
$$

There is a natural 1-1 correspondence between $e$-tuples of partitions $\pi=\left(\pi_{1}, \ldots, \pi_{e}\right)$ $\vdash r$ of $r$ and equivalence classes of $e$-symbols of rank $r$, as follows: by adding zeros we may assume that all $\pi_{i}=\left(\pi_{i 1} \leq \ldots \leq \pi_{i m}\right)$ have the same number of parts. It is easily verified that $S(\pi)=\left(S_{1}, \ldots, S_{e}\right)$, with $S_{i}:=\left(\pi_{i 1}, \pi_{i 2}+1, \ldots, \pi_{i m}+m-1\right)$ for $1 \leq i \leq e$, has indeed rank $r$, and is well defined up to equivalence.

Let $\left(v ; u_{1}, \ldots, u_{e}\right)$ be indeterminates over $\mathbb{Q}$. For an $e$-symbol $S$ we define

$$
f_{S}:=(-1)^{c(S)} \frac{(v-1)^{r} \prod_{i=1}^{e} u_{i}^{r} \cdot \prod_{i=1}^{e} \prod_{j=i}^{e} \prod_{s \in S_{i}} \prod_{\substack{t \in S_{j} \\ s>t \text { if } i=j}}\left(v^{s} u_{i}-v^{t} u_{j}\right)}{v^{a(S)} \prod_{i<j}\left(u_{i}-u_{j}\right)^{m} \cdot \prod_{i, j=1}^{e} \prod_{s \in S_{i}} \prod_{k=1}^{s}\left(v^{k} u_{i}-u_{j}\right)},
$$

where

$$
c(S):=\left(\begin{array}{l}
e \\
2
\end{array}\right)\left(\begin{array}{c}
m \\
2
\end{array}\right)+r(e-1), \quad \text { and } a(S):=\sum_{i=1}^{m-1}\left(\begin{array}{c}
e i \\
2
\end{array}\right)
$$

(see [27, (5.12)]). It can be checked that the rational function $f_{S}$ only depends on the equivalence class of the $e$-symbol $S$. We shall also write $f_{\pi}$ for $f_{S}$ with $S=S(\pi)$.

The following connection to the imprimitive complex reflection group $G(e, 1, r) \cong$ $C_{e} \imath \mathfrak{S}_{r}$ will be important for us. The irreducible complex characters of the wreath product $G(e, 1, r)$ can be parametrized by $e$-tuples of partitions $\left(\pi_{1}, \ldots, \pi_{e}\right)$ of $r$ (see for example [27, (2A)]), hence by equivalence classes of $e$-symbols of rank $r$. Now let $\mathcal{H}=\mathcal{H}(W, \mathbf{u})$ denote the cyclotomic Hecke algebra for $W=G(e, 1, r)$ with parameters $\mathbf{u}=\left(v ; u_{1}, \ldots, u_{e}\right)$. This carries a canonical symmetrizing form. By the main result of Geck-Iancu-Malle [19] the Schur element (with respect to this form) of the irreducible character of $\mathcal{H}$ indexed by the multipartition $\left(\pi_{1}, \ldots, \pi_{e}\right) \vdash r$ is $f_{S}^{-1}$, where $S=S\left(\pi_{1}, \ldots, \pi_{e}\right)$ (see Conjecture 2.20 in [27]).

In particular, specializing $v$ to 1 and $u_{j}$ to the eth roots of unity $\zeta_{j}:=\exp (2 \pi i j / e)$ we obtain

$$
f_{S}\left(1 ; \zeta_{1}, \ldots, \zeta_{e}\right)=\frac{d_{S}}{|G(e, 1, r)|}=\frac{d_{S}}{e^{r} r !},
$$

where $d_{S}$ denotes the degree of the irreducible character of $G(e, 1, r)$ indexed by $S$.

For later use let's record the following special cases. If $r=1$, so $G(e, 1, r)$ is the cyclic group $C_{e}$, then a multipartition $\pi=\left(\pi_{1}, \ldots, \pi_{e}\right) \vdash r$ is uniquely determined 
by the unique $i$ such that $\pi_{i}=(1)$. The corresponding $e$-symbol $S$ has $S_{i}=(1)$, $S_{j}=(0)$ for $j \neq i$, and

$$
f_{S}=\prod_{j \neq i} \frac{u_{j}}{u_{j}-u_{i}}
$$

(compare [5, Bem. 2.4])

More generally the $e$-symbols with $S_{i}=(r), S_{j}=(0)$ for $j \neq i$ parametrize linear characters $\varphi_{i}$ of $G(e, 1, r)$, for $1 \leq i \leq e$. Evaluation of the defining formula shows that then

$$
f_{S}=\prod_{k=1}^{r}\left(\frac{v-1}{v^{k}-1} \cdot \prod_{j \neq i} \frac{u_{j}}{u_{j}-v^{k-1} u_{i}}\right) .
$$

7.3. $d$-Harish-Chandra series and cyclotomic Hecke algebras. The blocks of finite groups of Lie type are closely related to so-called $d$-Harish-Chandra series. Let $G$ be as above, the group of fixed points of a simple algebraic group $\mathbf{G}$ under a Frobenius map. For any $d \in \mathbb{N}$, there is a notion of a $d$-split Levi subgroup $\mathbf{L}$ of $\mathbf{G}$ (an $F$-stable Levi subgroup of $\mathbf{G}$ ), and of a $d$-cuspidal unipotent character of $L:=\mathbf{L}^{F}$; see for example [6]. A pair $(L, \lambda)$ consisting of a $d$-split Levi subgroup $L \leq G$ with a $d$-cuspidal unipotent character $\lambda \in \mathcal{E}(L)$ of $L$ is called a $d$-cuspidal pair. Its relative Weyl group is then defined as

$$
W_{G}(L, \lambda):=\mathrm{N}_{G}(\mathbf{L}, \lambda) / L .
$$

By Broué-Malle-Michel [7, Thm. 3.2], the set of unipotent characters of $G$ admits a natural partition

$$
\mathcal{E}(G)=\coprod_{(L, \lambda) / \sim} \mathcal{E}(G,(L, \lambda))
$$

into $d$-Harish-Chandra series $\mathcal{E}(G,(L, \lambda))$, where $(L, \lambda)$ runs over the $d$-cuspidal pairs in $G$ modulo conjugation. Furthermore, for each $d$-cuspidal pair $(L, \lambda)$, there is a bijection

$$
\rho(L, \lambda): \mathcal{E}(G,(L, \lambda)) \stackrel{1-1}{\longrightarrow} \operatorname{Irr}\left(W_{G}(L, \lambda)\right)
$$

between its $d$-Harish-Chandra series and the irreducible characters of its relative Weyl group $W_{G}(L, \lambda)$. The degree polynomials are then given by the following $d$-analogue of Howlett-Lehrer-Lusztig theory:

Theorem 7.3. Let $(L, \lambda)$ be a d-cuspidal pair of $G$. Then for any $\varphi \in \operatorname{Irr}\left(W_{G}(L, \lambda)\right)$ there exists a rational function $D_{\varphi}(x) \in \mathbb{Q}(x)$ with zeros and poles only at roots of unity or zero, but not at primitive dth roots of unity, satisfying

$$
\operatorname{Deg}(\gamma)= \pm|G: L|_{x^{\prime}} D_{\rho(L, \lambda)(\gamma)} \operatorname{Deg}(\lambda) \quad \text { for all } \gamma \in \mathcal{E}(G,(L, \lambda))
$$

and

$|G: L|_{x^{\prime}} D_{\varphi} \equiv\left|W_{G}(L): W_{G}(L, \lambda)\right| \varphi(1) \quad\left(\bmod \Phi_{d}(x)\right) \quad$ for all $\varphi \in \operatorname{Irr}\left(W_{G}(L, \lambda)\right)$.

See [29, Thm. 4.2] and the references given there. In fact, the $D_{\varphi}(x)$ are inverses of Schur elements of a cyclotomic Hecke algebra attached to $W_{G}(L, \lambda)$ with respect to its canonical symmetrizing form. For example, if $W_{G}(L, \lambda) \cong G(e, 1, r)$, then $D_{\varphi}(x)$ is a suitable specialization of $f_{\varphi}(\mathbf{u})$ as defined above. 
We first determine for which parameters $\left(u_{1}, \ldots, u_{e}\right)$ all Schur elements of the cyclotomic Hecke algebra for the cyclic group $G(e, 1,1)$ are equal:

Lemma 7.4. Let $K$ be a field of characteristic 0 and $u_{i} \in K^{\times}, 1 \leq i \leq e$, pairwise distinct. If $\prod_{\substack{k=1 \\ k \neq i}}^{e} u_{k} /\left(u_{k}-u_{i}\right)$ is independent of $i$, then there exists $y \in K^{\times}$with

$$
\left\{u_{i} \mid 1 \leq i \leq e\right\}=\left\{\zeta^{i} y \mid 1 \leq i \leq e\right\}
$$

where $\zeta \in K$ is a primitive eth root of unity.

Proof. Equivalently we may assume that $u_{i} \prod_{k \neq i}\left(u_{i}-u_{k}\right)$ is independent of $i$. Thus, with $f:=\prod_{k=1}^{e}\left(x-u_{k}\right) \in K\left(u_{1}, \ldots, u_{e}\right)[x]$ and ' denoting the derivative with respect to $x$,

$$
u_{i} f^{\prime}\left(u_{i}\right)=u_{i} \prod_{\substack{k=1 \\ k \neq i}}^{e}\left(u_{i}-u_{k}\right)=: c
$$

is independent of $i$. That is, $u_{1}, \ldots, u_{e}$ are zeros of the polynomial $g:=x f^{\prime}-c$ of degree $e$, so $g=b \prod_{k=1}^{e}\left(x-u_{k}\right)=b f$ for some $b \in K$. Writing $f=\sum_{j=0}^{e} a_{j} x^{j}$ we have $j a_{j}=b a_{j}$ for $j=1, \ldots, e$. Since $a_{e}=1$ we conclude that $b=e$, and thus $a_{j}=0$ for $j=1, \ldots, e-1$. The claim follows.

The following result will allow us to show the existence of different height zero degrees in blocks of classical groups, that is, groups of type $A_{n}, B_{n}, C_{n}, D_{n},{ }^{2} A_{n}$ or ${ }^{2} D_{n}$.

Proposition 7.5. Let $G=G(q)$ be quasi-simple of classical type. Let $(L, \lambda)$ be a d-cuspidal pair in $G$. Assume that $W_{G}(L, \lambda) \neq 1$. Then there exist unipotent characters $\gamma_{1}, \gamma_{2} \in \mathcal{E}(G,(L, \lambda))$ with the following properties:

(a) $\gamma_{1}(1) \neq \gamma_{2}(1)$, and

(b) $\rho(L, \lambda)\left(\gamma_{i}\right) \in \operatorname{Irr}\left(W_{G}(L, \lambda)\right)$ are linear characters.

More precisely, $\gamma_{1}(1)_{q}<\gamma_{2}(1)_{q}$, or $q=2, d=2$ and $G$ is of type $D_{n}$ or ${ }^{2} D_{n}$.

Proof. We will show that $W_{G}(L, \lambda)$ has linear characters $\varphi_{1}, \varphi_{2}$ such that $D_{\varphi_{1}}(q) \neq$ $D_{\varphi_{2}}(q)$. The claim then follows from Theorem 7.3. In groups of classical type, there are three essentially different possibilities for the structure of the relative Weyl group (see [5, (3B)]). First, $W_{G}(L, \lambda)$ could be a symmetric group $\mathfrak{S}_{n}$. This happens if and only if either $G=\mathrm{SL}_{n}(q)$ and $d=1$, or $G=\mathrm{SU}_{n}(q)$ and $d=2$. In both cases, all of $\mathcal{E}(G)$ is just one $d$-Harish-Chandra series and we may take the trivial and the Steinberg character, which correspond to the two linear characters of $\mathfrak{S}_{n}$ and have distinct degrees.

The second possibility is that $W_{G}(L, \lambda) \cong G(m, 1, r)$ for some $m \geq 2$. This occurs for all other $d$-Harish-Chandra series $\mathcal{E}(G,(L, \lambda))$ in classical groups for which $\lambda$ is not parametrized by a so-called degenerate symbol. Let $\varphi_{i}$ denote the linear character of $G(m, 1, r)$ parametrized by the multipartition $\left(\pi_{1}, \ldots, \pi_{m}\right)$ with $\pi_{i}=(r)$. According to (11) we have $D_{\varphi_{i}}=c f_{i}^{-1}(\mathbf{q})$, where

$$
f_{i}\left(v, u_{1}, \ldots, u_{m}\right):=u_{i}^{r} \prod_{\substack{j=1 \\ j \neq i}}^{m} \prod_{k=0}^{r-1}\left(v^{k} u_{i}-u_{j}\right)
$$


for some nonzero $c$ not depending on $i$, and the parameters $\mathbf{q}$ are certain powers of $q$, up to sign, as follows (see [5, Bem. 2.10, 2.14, 2.19]):

(I) for $G=\mathrm{SL}_{n}(q), d \neq 1$, we have $m=d$ and

$$
\mathbf{q}=\left(q^{d} ; 1, q^{b_{1} d+1}, q^{b_{2} d+2}, \ldots, q^{b_{d-1} d+d-1}\right),
$$

$\left(\mathrm{I}^{\prime}\right)$ for $G=\mathrm{SU}_{n}(q), d \neq 2$, we have $m=d^{*}$, and $\mathbf{q}$ is obtained from the parameters in case (I) for $d^{*}$ by replacing $q$ by $-q$,

(II) for $G$ of type $B_{n}, C_{n}, D_{n},{ }^{2} D_{n}$ and $d$ odd we have $m=2 d, e=d$ and

$$
\mathbf{q}=\left(q^{e} ; 1, q^{b_{1} e+1}, \ldots, q^{b_{e-1} e+e-1},-q^{b_{e} e}, \ldots,-q^{b_{2 e-1} e+e-1}\right),
$$

(II') for $G$ of type $B_{n}, C_{n}, D_{n},{ }^{2} D_{n}$ and $d \equiv 2(\bmod 4)$ we have $m=d$, and $\mathbf{q}$ is obtained from the parameters in case (II) for $d^{*}$ by replacing $q$ by $-q$,

(III) for $G$ of type $B_{n}, C_{n}, D_{n},{ }^{2} D_{n}$ and $d \equiv 0(\bmod 4)$ we have $m=d, e=d / 2$ and

$$
\mathbf{q}=\left(-q^{e} ; 1, q^{b_{1} e+1}, \ldots, q^{b_{e-1} e+e-1},-(-1)^{b_{e}} q^{b_{e} e}, \ldots,-(-1)^{b_{2 e-1}} q^{b_{2 e-1} e+e-1}\right),
$$

where the $b_{i}$ are nonnegative integers which are determined by $\lambda$. Here, for $d \in \mathbb{N}$, $d^{*}$ is defined by

$$
d^{*}:= \begin{cases}2 d & \text { if } d \text { is odd }, \\ d / 2 & \text { if } d \equiv 2 \quad(\bmod 4), \\ d & \text { if } d \equiv 0 \quad(\bmod 4) .\end{cases}
$$

Note that it can never happen that $v^{k} u_{i}-u_{j}=0$ for the above choices of parameters. Furthermore, we claim that there is at least one $i$ with $\left|u_{i}\right|>1$. Indeed, otherwise we are necessarily in cases (II) or (II') and $e=1$. But then, since $\lambda$ is not parametrized by a degenerate symbol, $b_{1}>0$ by the definition of the $b_{i}$ in [5. (2B)], so $\left|u_{2}\right|>1$, a contradiction.

By our above reductions it suffices now to show that not all $f_{i}(\mathbf{q})$ are equal. For this, we estimate the $q$-power in $f_{i}(\mathbf{q})$ for two choices of $i$. For $i=1$ we have $u_{1}=1$, and $v^{k} u_{1}-u_{j}$ is at most divisible by the $q$-power $u_{j}$ (at least when $q$ is odd), and not divisible by $q$ if $k=0$, so $f_{1}(\mathbf{q})$ is at most divisible by the $q$-power $\prod_{j=2}^{m} u_{j}^{r-1}$. Now let $i$ be such that $\left|u_{i}\right|$ is maximal among the $\left\{u_{1}, \ldots, u_{m}\right\}$. Then $v^{k} u_{i}-u_{j}$ is divisible by at least the $q$-power $u_{j}$, so $f_{i}(\mathbf{q})$ is at least divisible by

$$
u_{i}^{r} \prod_{\substack{j=1 \\ j \neq i}}^{m} u_{j}^{r}=\prod_{j=1}^{m} u_{j}^{r} .
$$

By our above observation we have $\left|u_{i}\right| \geq q$, so $f_{1}(\mathbf{q}) \neq f_{i}(\mathbf{q})$, as claimed.

If $q$ is even, then $v^{k} u_{1}-u_{j}$ is divisible by $2 u_{j}$ if $v^{k} u_{1}=-u_{j}$. For fixed $j$, this can only happen for at most one value of $k$, and only when $j \equiv 1+e(\bmod 2 e)$ and we are in cases (II), (II') or (III). Thus, we get an additional factor at most 2 in the $q$-part of $f_{1}(\mathbf{q})$. It follows that the $q$-parts of $f_{1}(\mathbf{q})$ and $f_{i}(\mathbf{q})$ can only agree if $\left|u_{i}\right|=q=2$ and all other $u_{j}$ have absolute value 1 . Thus $e=1$, we are in cases (II) or $\left(\mathrm{II}^{\prime}\right)$ and $\mathbf{q}=(q ; 1,-q)$. But then

$$
f_{1}(\mathbf{q})=\prod_{k=0}^{r-1}\left(q^{k}+q\right) \neq \prod_{k=0}^{r-1}\left(q^{k+2}+q\right)
$$

(or the same with $q$ replaced by $-q$ ). 
Finally, we consider the case where $\lambda$ is parametrized by a degenerate symbol, which can only happen in types $D_{n}$ and ${ }^{2} D_{n}$. Then $W_{G}(L, \lambda) \cong G(2 m, 2, r)$, for some $m \geq 1$. We denote by $\psi_{1}, \ldots, \psi_{m}$ the $m$ distinct linear characters contained in the restrictions of the linear characters $\varphi_{1}, \ldots, \varphi_{2 m}$ from $G(2 m, 1, r)$ to its normal subgroup $G(2 m, 2, r)$ of index 2. Evaluation of [27, (5.12)] shows that $D_{\psi_{i}}=$ $\tilde{c} g_{i}^{-1}(\mathbf{q})$ for some constant $\tilde{c}$, where

$$
g_{i}\left(v, u_{1}, \ldots, u_{m}\right):=u_{i}^{r} \prod_{\substack{j=1 \\ j \neq i}}^{m} \prod_{k=0}^{r-1}\left(v^{k} u_{i}-u_{j}\right) \quad(1 \leq i \leq m),
$$

and the $\mathbf{q}$ are certain powers of $q$, up to sign, as follows (see [5, Bem. 2.16, 2.19]):

(IV) for $G$ of type $D_{n},{ }^{2} D_{n}$ and $d$ odd we have $m=e=d$ and

$$
\mathbf{q}=\left(q^{e} ; 1, q^{2 b_{1} e+2}, \ldots, q^{2 b_{e-1} e+2 e-2}\right),
$$

$\left(\mathrm{IV}^{\prime}\right)$ for $G$ of type $D_{n},{ }^{2} D_{n}$ and $d \equiv 2(\bmod 4)$ we have $m=d^{*}$, and $\mathbf{q}$ is obtained from the parameters in case (IV) for $d^{*}$ by replacing $q$ by $-q$,

(V) for $G$ of type $D_{n},{ }^{2} D_{n}$, and $d \equiv 0(\bmod 4)$ we have $m=e=d / 2$ and

$$
\mathbf{q}=\left(-q^{e} ; 1, q^{b_{1} d+2}, \ldots, q^{b_{e-1} d+d-2}\right) .
$$

Clearly, unless $d=1$, we can argue as before to conclude that $g_{1}(\mathbf{q}) \neq g_{i}(\mathbf{q})$ for a suitable index $i$. If $d=1$, then $W_{G}(L, \lambda) \cong G(2,2, r)$ is the Weyl group of type $D_{r}$, and we are in the principal 1-series of $G$. Here instead we take the trivial and the Steinberg character, which have distinct degree.

7.4. Unipotent blocks. After these combinatorial preparations we are ready to investigate unipotent blocks of groups of Lie type $G=G(q)$; here a $p$-block of $G$ is called unipotent if it contains at least one unipotent character of $G$.

Theorem 7.6. Let $\mathbf{G}$ be a simple algebraic group of simply connected type, $F$ : $\mathbf{G} \rightarrow \mathbf{G}$ a Frobenius map with group of fixed points $G=\mathbf{G}^{F}$. Let $B$ be a unipotent p-block of $G$, where $p$ is not the defining characteristic $r$ of $\mathbf{G}$. Then either $B$ is of defect 0 , or $B$ contains two height 0 characters of different degrees. Moreover, these two degrees have different $r$-parts, unless possibly if $r=2$.

Proof. First assume that $p$ is a good prime for $G$, odd, and not equal to 3 when $G$ is not of type ${ }^{3} D_{4}$. Then by Cabanes-Enguehard [10, Thm. 22.9] the intersections of unipotent $p$-blocks with $\mathcal{E}(G)$ are just the $d$-Harish-Chandra series, where $d$ is the multiplicative order of $q$ modulo $p$. Let $B$ be a unipotent $p$-block corresponding to the $d$-Harish-Chandra series of the $d$-cuspidal pair $(L, \lambda)$. If $L=G$, so $\lambda$ is a $d$ cuspidal character of $G$, then the defect group of $B$ is trivial by [10, Thm. 22.9(ii)], whence $B$ is of defect 0 .

If $L<G$, then $W_{G}(L, \lambda) \neq 1$. Now

$$
\operatorname{Deg}(\gamma)= \pm|G: L|_{x^{\prime}} D_{\varphi} \operatorname{Deg}(\lambda)
$$

for $\gamma \in \mathcal{E}(G,(L, \lambda))$, where $\varphi:=\rho(L, \lambda)(\gamma)$, and

$$
|G: L|_{q^{\prime}} D_{\varphi}(q) \equiv\left|W_{G}(L): W_{G}(L, \lambda)\right| \varphi(1) \quad\left(\bmod \Phi_{d}(q)\right)
$$

for $\varphi \in \operatorname{Irr}\left(W_{G}(L, \lambda)\right)$, by Theorem 7.3. As $p$ divides $\Phi_{d}(q)$ by definition, this implies the same congruence $(\bmod p)$. By the description in [10, Thm. 22.9(ii)], some unipotent character in $B$ is of height zero. This shows that the unipotent characters in $B$ of height 0 are precisely those $\gamma \in \mathcal{E}(G,(L, \lambda))$ with $\varphi=\rho(L, \lambda)(\gamma)$ 
of degree prime to $p$, for example the linear characters of $W_{G}(L, \lambda)$. For $G$ of classical type it is shown in Proposition 7.5 that not all unipotent characters in $B$ parametrized by linear characters of $W_{G}(L, \lambda)$ have the same degree.

If $G$ is of exceptional type and $W_{G}(L, \lambda)$ is cyclic, we may invoke Lemma 7.4 together with the parameters in [5, Tab. 8.1] to conclude the proof. The relative Weyl groups $W_{G}(L, \lambda)$ for exceptional groups which are noncyclic are listed in [5. Tab. 3.6] and [7, Tab. 1]. It is easy to check that these have two distinct character degrees prime to $p$, for all primes $p$ which are good for $G$. But then the corresponding unipotent degrees must be distinct by Proposition [7.2 and of height 0 by Theorem 7.3 .

Next, if $G$ is of classical type and $p=2$, then all unipotent characters of $G$ lie in the principal $p$-block of $G$, by [10, Th. 21.14]. Here, the trivial character and the Steinberg character have $p$-height 0 and different degrees.

It remains to consider the case where $G$ is of exceptional type and $p$ is a bad prime for $G$ (including the case of ${ }^{3} D_{4}$ with $p=3$ ). There is no bad prime for ${ }^{2} B_{2}$. The 2-blocks for ${ }^{2} G_{2}$ and the 3-blocks of ${ }^{2} F_{4}$ have been determined by Fong [18, resp. Malle [26, Bem. 1]: unipotent characters lie either in the principal block or are of defect zero. In the principal block the trivial and the Steinberg characters have different degrees.

TABLE 1. Nonprincipal $p$-blocks of positive defect for bad $p$

\begin{tabular}{rrllll}
\hline$G$ & $(p, d)$ & $L$ & $\lambda$ & $W_{G}(L, \lambda)$ & $\gamma_{1}, \gamma_{2}$ \\
\hline$F_{4}$ & $(3,1)$ & $2^{2} \cdot B_{2}$ & 1 & $C_{2}$ & $B_{2}, 1 ; B_{2}, \epsilon$ \\
$E_{6}$ & $(3,1)$ & $1^{2} \cdot D_{4}$ & $\zeta_{1}$ & $\mathfrak{S}_{3}$ & \\
& $(3,2)$ & $2 . A_{5}$ & $\xi$ & $C_{2}$ & $\varphi_{64,4} ; \varphi_{64,13}$ \\
$E_{7}$ & $(2,1)$ & $1 . E_{6}$ & $E_{6}[\theta]$ & $C_{2}$ & $E_{6}[\theta], 1 ; E_{6}[\theta], \epsilon$ \\
& $(3,1)$ & $1^{3} \cdot D_{4}$ & $\zeta_{1}$ & $W\left(B_{3}\right)$ & \\
$E_{8}$ & $(2$ or 5,1$)$ & $1^{2} \cdot E_{6}$ & $E_{6}[\theta]$ & $W\left(G_{2}\right)$ & $E_{6}[\theta], \varphi_{1,0} ; E_{6}[\theta], \varphi_{1,6}$ \\
& $(3$ or 5,1$)$ & $1^{4} \cdot D_{4}$ & $\zeta_{1}$ & $W\left(F_{4}\right)$ & \\
& $(3$ or 5,1$)$ & $1 . E_{7}$ & $E_{7}[\xi]$ & $C_{2}$ & $E_{7}[\xi], 1 ; E_{7}[\xi], \epsilon$ \\
& $(5,4)$ & $4^{2} . D_{4}$ & $\xi_{1}, \ldots, \xi_{4}$ & $G_{8}$ & \\
\hline
\end{tabular}

For the other types of exceptional groups, we use the description of unipotent blocks for bad primes $p$ obtained by Enguehard [16, Th. A]. Here, again any unipotent $p$-block is either of defect 0 , or it contains at least one nontrivial $d$-HarishChandra series. According to loc. cit. and the tables in [16, pp. 347-358], the nonprincipal unipotent blocks not of defect zero are as listed in Table 1 (up to Ennola duality and algebraic conjugacy; the notation is as in loc. cit.). In each case either the relative Weyl group has two distinct character degrees prime to $p$, in which case we may conclude as above, or we list two unipotent characters $\gamma_{1}, \gamma_{2}$ in the corresponding $d$-Harish-Chandra series which are of $p$-height 0 and have distinct $r$-parts in their degrees (see [7, Tab. 2] for the list of $d$-Harish-Chandra series and [11, Ch. 13] for the degrees of unipotent characters).

This completes the proof of Theorem 7.6. Note that the results hold even when the finite group $G$ is not perfect, or even solvable. 
We now extend the result to unipotent blocks of arbitrary finite connected reductive groups.

Theorem 7.7. Let $\mathbf{G}$ be a connected reductive group with a Frobenius map $F$ : $\mathbf{G} \rightarrow \mathbf{G}$ and group of fixed points $G:=\mathbf{G}^{F}$. Let $B$ be a unipotent $p$-block of $G$, where $p$ is not the defining characteristic $r$ of $\mathbf{G}$. Then either $B$ is of central defect, and all characters of $B$ have the same degree, or $B$ contains two height 0 characters of different degrees. Moreover, these two degrees have different r-parts unless possibly if $r=2$.

Proof. The derived group $[\mathbf{G}, \mathbf{G}]$ is semisimple, hence a central product $\mathbf{G}_{1} \circ \ldots \circ \mathbf{G}_{r}$ of simple algebraic groups. We assume the $\mathbf{G}_{i}$ ordered such that $\mathbf{G}_{1}, \ldots, \mathbf{G}_{s}$, for some $s \leq r$, is a system of representatives for the $F$-orbits on $\left\{\mathbf{G}_{1}, \ldots, \mathbf{G}_{r}\right\}$. Then, $G^{\prime}:=[\mathbf{G}, \mathbf{G}]^{F}$ is a central product $G_{1} \circ \ldots \circ G_{s}$ of groups, with $G_{i} \cong \mathbf{G}_{i}^{F^{m_{i}}}$, where $m_{i}$ is the size of the $F$-orbit of $\mathbf{G}_{i}$. Note that, in general, $G^{\prime}$ will be larger than the commutator subgroup of $G$. Modulo $Z([\mathbf{G}, \mathbf{G}])^{F}=Z\left(G^{\prime}\right)$ we obtain a direct product

$$
\bar{G}:=G^{\prime} / Z([\mathbf{G}, \mathbf{G}])^{F} \cong \bar{G}_{1} \times \ldots \times \bar{G}_{s},
$$

where $\bar{G}_{i}:=G_{i} /\left(G_{i} \cap Z([\mathbf{G}, \mathbf{G}])^{F}\right)$. Now let $B$ be a unipotent $p$-block of $G$. Since unipotent characters restrict irreducibly to the $F$-fixed points of the derived group [25] $B$ covers a unique block $B^{\prime}$ of $G^{\prime}$. Furthermore unipotent characters have the center in their kernel, so the same holds for unipotent blocks. Thus $B^{\prime}$ corresponds to a unique block $\bar{B}$ of the direct product $\bar{G}$. This is a direct product $\bar{B}=\bar{B}_{1} \times \ldots \times \bar{B}_{s}$ of blocks $\bar{B}_{i}$ of $\bar{G}_{i}$.

Now assume that one of the $\bar{B}_{i}$ is not of defect 0 for $\bar{G}_{i}$. Since $\bar{G}_{i}$ is a central factor group of a group as in Theorem 7.6, $\bar{B}_{i}$ then contains two height 0 unipotent characters of different degrees, with different $r$-parts if $r=2$. Thus, the same is true for $\bar{B}$, hence also for $B^{\prime}$. By the above-mentioned irreducibility of restrictions, this then also holds for $B$.

On the other hand, if all $\bar{B}_{i}$ are of defect 0 , then so is $\bar{B}$, so $B^{\prime}$ is of central defect, contained in $Z([\mathbf{G}, \mathbf{G}])^{F}$. But $Z([\mathbf{G}, \mathbf{G}]) \subseteq Z(\mathbf{G})$ as $\mathbf{G}=[\mathbf{G}, \mathbf{G}] Z(\mathbf{G})$, so the block $B$ is also of central defect in $G$, as claimed. Moreover, as each $\bar{B}_{i}$ contains a unique ordinary character, we also have $\operatorname{Irr}\left(B^{\prime}\right)=\left\{\chi^{\prime}\right\}$ for some ordinary (unipotent) character $\chi^{\prime}$ of $G^{\prime}$. Since this is the restriction of an irreducible character of $G$, and $G / G^{\prime}$ is abelian, all characters of $G$ above $\chi^{\prime}$ have the same degree; hence the height zero conjecture holds for $B$ in this case.

Proposition 7.8. Let $G$ be a finite group, $N \triangleleft G$ a normal subgroup of index prime to $p$ with $G / N$ either cyclic or a Klein four group. Let $b$ be a $p$-block of $G$, and $b^{\prime}$ a p-block of $N$ lying below $b$. Then:

(a) $b$ and $b^{\prime}$ have isomorphic defect groups.

(b) Assume that $b^{\prime}$ has two height 0 characters $\chi_{1}, \chi_{2}$ of different degrees, and let $r$ be a prime for which the $r$-parts of $\chi_{1}(1), \chi_{2}(1)$ differ. If $\operatorname{gcd}(r, \mid G$ : $N \mid)=1$, then $b$ also has two height 0 characters of different degrees.

Proof. The first assertion is well known. For the second, let $\nu_{i}$ be a character of $b$ lying above $\chi_{i}$, for $i=1,2$. Since $p$ does not divide the index $|G: N|, \nu_{i}$ is again of height 0 . Furthermore, the assumption that $\operatorname{gcd}(r,|G: N|)=1$ and $G / N$ is cyclic or Klein four implies by Clifford theory that $\nu_{1}(1) \neq \nu_{2}(1)$. 


\section{Blocks of GRoups OF LiE TYPE}

Proposition 8.1. The assertion of Theorem 6.1 holds when $S$ is a simple group of Lie type and $p$ is the defining characteristic.

Proof. By the result of Humphreys 22 the covering group $G$ of $S$ has exactly one $p$-block of defect zero, consisting of the Steinberg character, and all other $p$-blocks are of full defect, in one-to-one correspondence with the irreducible characters of $Z(G)$. More precisely, two characters lie in the same $p$-block if and only if they have the same restriction to $Z(G)$. For the principal block, it is clear that there exist two height 0 characters of distinct degree (viz. the trivial character and at least one further nonlinear semisimple character). For the remaining blocks, some more work is needed. By the above we may now assume that $Z(G) \neq 1$, so in particular $p$ is odd for classical groups not of types $A_{n}$ or ${ }^{2} A_{n}$.

If $s \in G^{*}$ is semisimple, the corresponding semisimple characters $\chi_{s} \in \operatorname{Irr}(G)$ are of $p^{\prime}$-degree given by $\chi_{s}(1)=\left|G: \mathrm{C}_{G^{*}}(s)\right|_{p^{\prime}}$ (see for example [29, (2.1)]; note that $\mathrm{C}_{G^{*}}(s)$ is not necessarily connected). Furthermore, $\left.\chi_{s}\right|_{Z(G)}=\left.\chi(1) \theta\right|_{Z(G)}$ if $(T, \theta)$ lies in the rational series parametrized by $s$ by [29, Lemma 2.2]. So we are done if for any character $\nu \neq 1$ of $Z(G)$ we can find two semisimple elements $s_{1}, s_{2} \in G^{*}$ whose centralizer orders have different $p^{\prime}$-part and such that $\left.\chi_{s_{i}}\right|_{Z(G)}=\nu$.

TABLE 2. Tori and Zsigmondy primes for classical groups

\begin{tabular}{lccll}
\hline & $\left|T_{1}\right|$ & $\left|T_{2}\right|$ & $\ell_{1}$ & $\ell_{2}$ \\
\hline$A_{n}$ & $\left(q^{n+1}-1\right) /(q-1)$ & $q^{n}-1$ & $l(n+1)$ & $l(n)$ \\
${ }^{2} A_{n}(n \geq 2$ even $)$ & $\left(q^{n+1}+1\right) /(q+1)$ & $q^{n}-1$ & $l(2 n+2)$ & $l(n)$ \\
${ }^{2} A_{n}(n \geq 3$ odd $)$ & $\left(q^{n+1}-1\right) /(q+1)$ & $q^{n}+1$ & $l(n+1)$ & $l(2 n)$ \\
$B_{n}, C_{n}(n \geq 2$ even $)$ & $q^{n}+1$ & $\left(q^{n-1}+1\right)(q+1)$ & $l(2 n)$ & $l(2 n-2)$ \\
$B_{n}, C_{n}(n \geq 3$ odd $)$ & $q^{n}+1$ & $q^{n}-1$ & $l(2 n)$ & $l(n)$ \\
$D_{n}(n \geq 4$ even $)$ & $\left(q^{n-1}-1\right)(q-1)$ & $\left(q^{n-1}+1\right)(q+1)$ & $l(n-1)$ & $l(2 n-2)$ \\
$D_{n}(n \geq 5$ odd $)$ & $q^{n}-1$ & $\left(q^{n-1}+1\right)(q+1)$ & $l(n)$ & $l(2 n-2)$ \\
${ }^{2} D_{n}(n \geq 4)$ & $q^{n}+1$ & $\left(q^{n-1}+1\right)(q-1)$ & $l(2 n)$ & $l(2 n-2)$ \\
\hline
\end{tabular}

In Table 2 we have listed two maximal tori $T_{1}, T_{2}$ of $G$ for each type of classical group $G$ (by giving their orders, which determines them uniquely). Except for types $B_{n}, C_{n}$ with $n$ even this is Table 3.5 in Malle 28. We write $l(m)$ for a Zsigmondy prime divisor of $q^{m}-1$. Then $\left|T_{i}\right|$ is divisible by the Zsigmondy prime $\ell_{i}$ as indicated in the table, which exists unless $G$ is of type $A_{1}$, or $G$ is of type $A_{2},{ }^{2} A_{2}$ or $B_{2}$ and $i=2$. (Note that the case that $q=2$ and $G$ of type $A_{5}, A_{6},{ }^{2} A_{3}$ or ${ }^{2} A_{6}$ does not concern us here, since then the center of $G$ is trivial, and for ${ }^{2} A_{5}(2)=\mathrm{SU}_{6}(2)$ the claim can be checked using 12.) If $\left|T_{i}\right|$ is divisible by a Zsigmondy prime $\ell_{i}$, then there exist characters $\theta_{i} \in \operatorname{Irr}\left(T_{i}\right)$ of order divisible by $\ell_{i}$ with given restriction $\nu$ to $Z(G) \leq T_{i}$ (since $|Z(G)|$ is prime to $\ell_{i}$ ). The order of the centralizer $\left|\mathrm{C}_{G^{*}}\left(s_{i}\right)\right|$ of semisimple elements $s_{i} \in G^{*}$ corresponding to $\left(T_{i}, \theta_{i}\right)$ is then divisible by $\left|T_{i}\right|$. If both Zsigmondy primes exist, this yields two semisimple characters with given restriction to $Z(G)$ and of different degrees, since no centralizer is divisible by both Zsigmondy primes (for example since we may take $s_{1}$ to be regular), so we are done in this case.

So now assume that $G$ is of type $A_{1}, A_{2},{ }^{2} A_{2}$ or $B_{2}$. From the known character tables it can be seen that the group $\mathrm{SL}_{2}(q)$ has faithful irreducible characters of degrees $q+1,(q-1) / 2$ for $q \equiv 1(\bmod 4)$, and of degrees $q-1,(q+1) / 2$ for 
$7 \leq q \equiv 3(\bmod 4)$. The group $\mathrm{SL}_{3}(q), q \equiv 1(\bmod 3)$, has faithful irreducible characters of degrees $q^{2}+q+1$ and $(q-1)\left(q^{2}-1\right)$, the group $\mathrm{SU}_{3}(q), 2<q \equiv 2$ (mod 3), has faithful irreducible characters of degrees $q^{2}-q+1$ and $(q+1)\left(q^{2}-1\right)$, and the group $\mathrm{Sp}_{4}(q), q$ odd, has faithful irreducible characters of degrees $\left(q^{2}-1\right) / 2$ and $q^{4}-1$, which are clearly distinct and of $p$-height zero.

The only exceptional simply connected groups with nontrivial center are those of types $E_{6},{ }^{2} E_{6}$ and $E_{7}$. For these, we may argue as above using the maximal tori and Zsigmondy primes listed in Table 3 . The proof is complete.

TABLE 3 . Tori $T_{1}$ and $T_{2}$ for exceptional groups

\begin{tabular}{rccll}
\hline$G$ & $\left|T_{1}\right|$ & $\left|T_{2}\right|$ & $\ell_{1}$ & $\ell_{2}$ \\
\hline$E_{6}(q)$ & $\Phi_{12} \Phi_{3}$ & $\Phi_{9}$ & $l(12)$ & $l(9)$ \\
${ }^{2} E_{6}(q)$ & $\Phi_{18}$ & $\Phi_{12} \Phi_{6}$ & $l(18)$ & $l(12)$ \\
$E_{7}(q)$ & $\Phi_{18} \Phi_{2}$ & $\Phi_{14} \Phi_{2}$ & $l(18)$ & $l(14)$
\end{tabular}

We now turn to the nondefining primes for groups of Lie type.

According to the work of Broué-Michel [8], for any $p$-block $B$ of $G$ there exists a unique $G^{*}$-conjugacy class $[s]$ of semisimple $p^{\prime}$-elements of $G^{*}$, such that some irreducible representation of $B$ is in the rational Lusztig series attached to $[s]$. Let's write $\mathcal{E}_{p}(G, s)$ for the union of all $p$-blocks of $G$ associated with the class of the $p^{\prime}$ element $s \in G^{*}$. The blocks in $\mathcal{E}_{p}(G, 1)$ are called unipotent. More generally, if $\mathbf{G}$ is disconnected, then a block of $\mathbf{G}^{F}$ is called unipotent if it covers a unipotent block of $\left(\mathbf{G}^{\circ}\right)^{F}$. We need the following crucial result of Enguehard [17, Th. 1.6]:

Theorem 8.2 (Enguehard). Assume that $p$ is good for $\mathbf{G}$, and different from 3 if $F$ induces a triality automorphism on $\mathbf{G}$.

Let $s \in G^{*}$ be a semisimple $p^{\prime}$-element, and $B$ a p-block in $\mathcal{E}_{p}(G, s)$. Then there exists a reductive group $\mathbf{G}(s)$ defined over $\mathbb{F}_{r}$, with corresponding Frobenius map again denoted by $F$, and a unipotent p-block $b$ of $G(s):=\mathbf{G}(s)^{F}$, such that the defect groups of $B$ and $b$ are isomorphic and there is a height-preserving bijection $\operatorname{Irr}(B) \rightarrow \operatorname{Irr}(b)$. Here, $\mathbf{G}(s)^{\circ}$ is a group in duality with $C_{\mathbf{G}^{*}}(s)^{\circ}$, and $\mathbf{G}(s) / \mathbf{G}(s)^{\circ} \cong$ $C_{\mathbf{G}^{*}}(s) / C_{\mathbf{G}^{*}}(s)^{\circ}$.

In the case of $p=2$ for classical groups, he proves [17, Prop. 1.5]:

Theorem 8.3 (Enguehard). Assume that $G$ is of classical type in odd characteristic. Let $s \in G^{*}$ be a semisimple $2^{\prime}$-element. Then all 2-blocks in $\mathcal{E}_{2}(G, s)$ have defect group isomorphic to a Sylow 2-subgroup of $\mathrm{C}_{G^{*}}(s)^{\circ}$. If moreover $G$ is of type $B_{n}, C_{n}$ or $D_{n}$, then $\mathcal{E}_{2}(G, s)$ is a single 2-block.

Proposition 8.4. The assertion of Theorem 6.1 holds if $G$ is quasi-simple of Lie type.

Proof. By Proposition 8.1 we may assume that $p$ is not the defining characteristic for $G$, and by Proposition 9.4 we may assume that $S \neq^{2} F_{4}(2)^{\prime}$. Furthermore, by the remarks at the beginning of Section 7 we have that $G=\mathbf{G}^{F}$ for some simple, simply connected algebraic group $\mathbf{G}$ with Frobenius map $F: \mathbf{G} \rightarrow \mathbf{G}$. 
Let $B$ be a $p$-block of $G$ and $s \in G^{*}$ semisimple such that $B \subseteq \mathcal{E}_{p}(G, s)$ (see above). First assume that $s$ is not quasi-isolated in $G^{*}$; that is, $C_{G^{*}}(s)$ is a Levi subgroup of $G^{*}$. Then by the result of Bonnafé-Rouquier [10, Th. 10.1] the block $B$ is Morita-equivalent to a block $b \subseteq \mathcal{E}_{p}(L, 1)$, where $L$ is a Levi subgroup of $G$ in duality with $C_{G^{*}}(s)$, and Jordan decomposition gives a height-preserving bijection from $B$ to $b$. We may then conclude by Theorem 7.7 .

Next assume that $p$ is good for $G$, different from 3 if $G$ is of type ${ }^{3} D_{4}$. Then by Theorem 8.2 there is a group $\mathbf{G}(s)$ in duality with the centralizer $\mathbf{C}:=C_{\mathbf{G}^{*}}(s)$ of $s$ in $\mathbf{G}^{*}$ and a height-preserving bijection between $B$ and a unipotent block $b$ of $G(s):=\mathbf{G}(s)^{F}$ with the same defect group as B. By [4, Cor. 2.9] the order $a(s):=\left|\mathbf{C}: \mathbf{C}^{\circ}\right|$ of the component group of $\mathbf{C}$ is prime to the defining characteristic $r$ and divides the order of $s$. As $s$ is a $p^{\prime}$-element, this implies that $a(s)$ is prime to $p$ as well. Moreover, by loc. cit., $\mathbf{C} / \mathbf{C}^{\circ}$ is isomorphic to a subgroup of the fundamental group of $\mathbf{G}$, hence either cyclic or a Klein four group. Now let $b^{\prime}$ be a $p$-block of the normal subgroup $N:=\left(\mathbf{C}^{\circ}\right)^{F}$ of $\mathbf{C}^{F}=C_{G^{*}}(s)$ lying below $b$. We showed in Theorem 7.7 that any unipotent block of the connected group $N$ with nonabelian defect group contains two height 0 characters with degrees divisible by different powers of the defining prime $r$. Thus, Proposition 7.8 applies in this case and the claim follows.

Now assume that $p=2$ and $G$ is of classical type $B_{n}, C_{n}, D_{n}$ or ${ }^{2} D_{n}$. Then $\mathcal{E}_{2}(G, s)$ is a single 2-block by Theorem 8.3. By Jordan decomposition the character degrees in $\mathcal{E}_{2}(G, s)$ are obtained from those in $\mathcal{E}_{2}\left(C_{G^{*}}(s), 1\right)$ by multiplication with a common constant. If $C_{G^{*}}(s)^{\circ}$ is not a torus, the trivial character and the Steinberg character in $\mathcal{E}_{2}\left(C_{G^{*}}(s), 1\right)$ have distinct degrees prime to $p$ and the claim follows. On the other hand, if $C_{G^{*}}(s)^{\circ}$ is a torus, that is, $s$ is a regular element in $G^{*}$, then again by Theorem 8.3 of Enguehard the defect group of $B$ is isomorphic to a Sylow 2-subgroup of $\mathrm{C}_{G^{*}}(s)^{\circ}$, hence abelian. Moreover, by the result of Lusztig [25], all characters in $B$ have the same degree, whence $B$ satisfies the height zero conjecture.

Thus we may assume that $G$ is of exceptional type, $p$ is a bad prime and $s$ is quasi-isolated. There are no quasi-isolated elements for ${ }^{2} B_{2}$. The $p$-blocks for ${ }^{2} G_{2}$, $G_{2},{ }^{2} F_{4}$ and ${ }^{3} D_{4}$ have been determined by Fong [18, Hiss-Shamash 20, 21, Malle [26], Deriziotis-Michler [13, respectively. The claim can be easily checked from those results.

The remaining cases are the possible exceptions mentioned in the theorem.

\section{Alternating And SPORAdic Groups}

In order to prove our main result for the alternating groups, we first derive a similar statement for blocks of the symmetric group.

Recall that the irreducible characters of $\mathfrak{S}_{n}$ as well as the unipotent characters of $\mathrm{GL}_{n}(q)$, where $q$ is any prime power, are parametrized by partitions $\lambda \vdash n$. We write $\chi_{\lambda}$, resp. $\gamma_{\lambda}$, for the corresponding character of $\mathfrak{S}_{n}$, resp. of $\operatorname{GL}_{n}(q)$. The following important connection between their degrees is well known: $\chi_{\lambda}$ is obtained by specializing $q$ to 1 in the degree polynomial for $\gamma_{\lambda}$ (see for example the formula in [11, 13.8] and compare to the hook formula for $\left.\chi_{\lambda}(1)\right)$. This is sometimes referred to by saying that $\mathfrak{S}_{n}$ is 'the general linear group over the field with one element'.

Furthermore, $\chi_{\lambda}$ and $\chi_{\mu}$ for two partitions $\lambda, \mu \vdash n$ lie in the same $p$-block of $\mathfrak{S}_{n}$ if and only if $\lambda$ and $\mu$ have the same $p$-core, which in turn happens if and only if $\gamma_{\lambda}$ and $\gamma_{\mu}$ lie in the same $d$-Harish-Chandra series of $\operatorname{Irr}\left(\mathrm{GL}_{n}(q)\right)$, where $d=p$. Thus, 
the degrees of irreducible characters of $\mathfrak{S}_{n}$ in a fixed $p$-block are specializations at $q=1$ of degree polynomials of unipotent characters in a fixed $p$-Harish-Chandra series.

Let $S=\left(S_{1}, \ldots, S_{d}\right)$ be a $d$-symbol. A hook of $S$ is a pair $h=(s, t)$, where

$$
s \in S_{i}, \quad t \in\{0, \ldots, s\} \backslash S_{j}, \quad \text { with } j>i \text { if } s=t,
$$

for some $1 \leq i, j, \leq d$. We then also write $i(h):=i, j(h):=j$, and $l(h):=s-t$. For 1-symbols, that is, $\beta$-sets of partitions, this is just the usual notion of hook. We can now formulate the following relative hook formula for characters in a fixed $p$-block of a symmetric group which seems to be new:

Theorem 9.1. Let $p$ be a prime. Let $\pi \vdash n$ be a partition with $p$-core $\mu \vdash r$ and $p$ quotient $\left(\nu_{1}, \ldots, \nu_{p}\right) \vdash w$, with corresponding $p$-symbol $S$. Let $b_{i}$ denote the number of beads on the ith runner of the $p$-abacus diagram for $\mu$, and $c_{i}:=p b_{i}+i-1$. Then

$$
\chi_{\pi}(1)=\frac{n !}{r !} \cdot \frac{1}{\prod_{h \text { hook of } S}\left|p l(h)+c_{i(h)}-c_{j(h)}\right|} \cdot \chi_{\mu}(1)
$$

and

$$
\chi_{\pi}(1) / \chi_{\mu}(1) \equiv \psi_{\nu}(1) \quad(\bmod p),
$$

where $\psi_{\nu}$ denotes the irreducible character of $C_{p} ح \mathfrak{S}_{w}$ parametrized by $\nu$.

Proof. Let $\gamma$ be the unipotent character of $\mathrm{GL}_{n}(q)$ parametrized by $\pi$, for $q$ a prime power. Set $d:=p$. Then $\gamma$ lies in the $d$-Harish-Chandra series above $(L, \lambda)$, where $L \cong \mathrm{GL}_{r}(q) \times \mathrm{GL}_{1}\left(q^{d}\right)^{w}$, with $\lambda$ parametrized by $\mu \vdash r$ and $n=r+d w$. Let $S=\left(S_{1}, \ldots, S_{d}\right)$ be the $d$-symbol corresponding to $\left(\nu_{1}, \ldots, \nu_{d}\right)$. According to Theorem [7.3, 27, (2.19)] we have $\operatorname{Deg}(\gamma) / \operatorname{Deg}(\lambda)= \pm|G: L|_{q^{\prime}} D_{\rho(L, \lambda)(\gamma)}=$

$$
\pm \frac{\prod_{i=1}^{n}\left(q^{i}-1\right)}{\left(q^{d}-1\right)^{w} \prod_{i=1}^{r}\left(q^{i}-1\right)} \cdot \frac{(v-1)^{w} \prod_{i=1}^{d} u_{i}^{w} \cdot \prod_{i=1}^{d} \prod_{j=i}^{d} \prod_{s \in S_{i}} \prod_{\substack{t \in S_{j} \\ s>t \text { if } i=j}}\left(v^{s} u_{i}-v^{t} u_{j}\right)}{v^{a(S)} \prod_{i<j}\left(u_{i}-u_{j}\right)^{m} \cdot \prod_{i, j=1}^{d} \prod_{s \in S_{i}} \prod_{k=1}^{s}\left(v^{k} u_{i}-u_{j}\right)}
$$

with

$$
\left(v ; u_{1}, \ldots, u_{d}\right)=\left(q^{d} ; 1, q^{c_{2}}, \ldots, q^{c_{d}}\right)
$$

(see (I) in Sect.7.3 for the parameter values). By our above remarks, specialization at $q=1$ gives the corresponding character degrees for $\mathfrak{S}_{n}$. Note that the numerator and denominator of the expression for $\operatorname{Deg}(\gamma) / \operatorname{Deg}(\lambda)$ are indeed divisible by the same power of $(q-1)$, viz. $n+w+\left(\begin{array}{c}m e \\ 2\end{array}\right)$, so that the specialization makes sense. 
We obtain

$$
\begin{array}{r}
\frac{\chi_{\pi}(1)}{\chi_{\mu}(1)}= \pm \frac{n !}{r !} \cdot \frac{\prod_{i=1}^{d} \prod_{j=i}^{d} \prod_{s \in S_{i}} \prod_{\substack{t \in S_{j} \\
s>t \text { if } i=j}}\left(d(s-t)+c_{i}-c_{j}\right)}{\prod_{i<j}\left(c_{i}-c_{j}\right)^{m} \cdot \prod_{i, j=1}^{d} \prod_{s \in S_{i}} \prod_{k=1}^{s}\left(d k+c_{i}-c_{j}\right)} \\
= \pm \frac{n !}{r !} \cdot \frac{1}{\prod_{h \text { hook of } S}\left(d l(h)+c_{i(h)}-c_{j(h)}\right)}
\end{array}
$$

as claimed.

Now choose $q$ such that $q \equiv 1(\bmod p)$. Then we have

$$
\gamma(1) / \lambda(1) \equiv \pm \psi_{\nu}(1) \quad\left(\bmod \Phi_{d}(q)\right)
$$

by Theorem 7.3 and

$$
\gamma(1) / \lambda(1) \equiv \pm \chi_{\pi}(1) / \chi_{\mu}(1) \quad(\bmod q-1)
$$

by our observation above. As $p$ divides both $\Phi_{d}(q)=q^{d-1}+\ldots+1$ and $q-1$, the stated congruence follows.

Let's note the following special case of $p$-quotients $\left(\nu_{1}, \ldots, \nu_{p}\right) \vdash w$ such that the corresponding $p$-symbol $S$ has $S_{i}=(w), S_{j}=(0)$ for $j \neq i$. Since these correspond to linear characters of the relative Weyl group in $\mathrm{GL}_{n}(q)$, they parametrize characters of height 0 in $B$ by the congruence in Theorem 9.1. We obtain

$$
\chi_{\pi}(1)=\frac{n !}{p^{w} r ! w !} \cdot \prod_{k=0}^{w-1} \prod_{j \neq i}\left|p k+c_{i}-c_{j}\right|^{-1} \cdot \chi_{\mu}(1) .
$$

A $p$-block $B$ of $\mathfrak{S}_{n}$ labelled by a $p$-core $\mu \vdash n-w p$ is said to be of weight $w$. So $w$ denotes the number of $p$-hooks which must be removed from any partition $\pi$ indexing a character in $B$ to obtain its core $\mu$. The block is said to be self-dual if $\mu$ is a self-dual partition.

Proposition 9.2. Let $G=\mathfrak{S}_{n}, n \geq 5, p$ a prime, and $B$ a p-block of $G$. Then one of the following occurs:

(a) $B$ is of weight (and hence defect) 0 ,

(b) $p=2$ and $B$ is of weight 1 ,

(c) $p=3, B$ is of weight 1 and self-dual, or

(d) $B$ contains two height 0 characters of different degrees $d_{1}<d_{2}$, either both indexed by non-self-dual partitions or with $d_{2} \neq 2 d_{1}$.

Proof. We use the relative hook formula in (44) for the character degrees of $\mathfrak{S}_{n}$ for certain height 0 characters in $B$. We may assume that the weight $w$ of $B$ is positive. Let $\mu \vdash n-p w$ denote the $p$-core associated to $B$, let $0=e_{1}<e_{2} \ldots<e_{p}$ be the ordered set of the $c_{i}$ as in Theorem 9.1, and $f_{i}:=\prod_{k=0}^{w-1} \prod_{j \neq i}\left|p k+e_{i}-e_{j}\right|$, for $1 \leq i \leq p$. Note that by [35. Prop. 3.5] none of the partitions $\lambda_{i}$ corresponding to the $p$-quotients $S_{i}$ is self-dual, unless $w=1$, in which case at most one of them is. Clearly, $f_{p}>f_{p-1}$ unless $p=2$ and $w=1$ (which is case (b)), which yields two 
distinct height 0 degrees $d_{1}, d_{2}$. If both corresponding partitions are self-dual, then $w=1$. But by Theorem 9.1 we have $d_{i} \equiv \pm 1(\bmod p)$, and then $d_{1}=d_{2} / 2$ implies that $p=3$.

Corollary 9.3. Let $p$ be a prime, $B$ a p-block of $\mathfrak{A}_{n}$. Then one of the following holds:

(a) $B$ is of defect 0 ,

(b) $p=3, B$ is of weight 1 (hence with cyclic defect group $C_{3}$ ), self-dual, and all $\chi \in \operatorname{Irr}(B)$ have the same degree, or

(c) B contains two height 0 characters of different degrees.

In particular, the assertion of Theorem 6.1 holds when $S$ is an alternating group.

Proof. Let $B$ be a $p$-block of $\mathfrak{S}_{n}$, containing all characters $\chi_{\lambda}$ for which $\lambda$ has fixed $p$-core $\mu \vdash(n-p w)$. According to [35. Prop. 12.2], for example, if $w>0$, then $B$ covers a unique block $B_{1}$ of $\mathfrak{A}_{n}$. First assume that $p$ is odd. Let $\chi_{1}, \chi_{2} \in B$ be two height zero characters of different degrees, parametrized by non-self-dual partitions, according to Proposition 9.2. These restrict irreducibly to characters of $\mathfrak{A}_{n}$ in $B_{1}$ of height 0 . Similarly, if $\chi_{1}, \chi_{2} \in B$ have different degrees $d_{1}<d_{2}$ with $d_{2} \neq 2 d_{1}$, then the restrictions of $\chi_{1}, \chi_{2}$ to $\mathfrak{A}_{n}$ contain characters of $B_{1}$ of height 0 and of different degrees.

If $p=3, B$ is of weight 1 and self-dual, then two characters of $B$ have the same irreducible restriction and one splits into two constituents for $\mathfrak{A}_{n}$. We obtain a block $B_{1}$ with defect group of order 3 and three equal character degrees.

For $p=2$, restriction of characters from $G$ to $B_{1}$ either preserves heights or decreases it, by [35, Prop. 12.5]. Thus, we may conclude by Proposition 9.2 unless $w=1$. Here, the two irreducible characters in $B$ have the same restriction to $\mathfrak{A}_{n}$, so $B_{1}$ is a block with a unique ordinary character, that is, a block of defect zero.

Note that case (b) of Corollary 9.3 occurs if and only if there is a self-dual 3-core for $n-3$. The conditions for this to occur have been worked out in [2, Lemma 3.1].

The block distribution of the faithful characters of $2 . \mathfrak{A}_{n}$ is known in general, but we have not been able to come up with a corresponding extension of the character formula in Theorem 9.1, mainly because there does not seem to be a suitable $q$ analogue of the spin covers $2 . \mathfrak{S}_{n}$. It can be checked from the known character tables that the assertion of Theorem 6.1 remains true for the faithful blocks of $2 . \mathfrak{A}_{n}$ when $n \leq 13$.

We complete our investigation of blocks of quasi-simple groups by showing:

Proposition 9.4. The assertion of Theorem 6.1 holds when $S$ is sporadic or a simple group of Lie type with exceptional Schur multiplier, or $S={ }^{2} F_{4}(2)^{\prime}$.

Proof. The ordinary character tables of all quasi-simple groups such that $S$ is as in the assumption are contained in the Atlas [12. From this, or using the electronic tables available in GAP, it can be checked that whenever $B$ is a $p$-block of $G$ with all height zero characters of the same degree, then the defect group satisfies $|D| \leq p^{2}$, hence must be abelian.

\section{0. $p$-Solvable Groups}

Our main result in this section is to reduce the study of EHZD blocks of general $p$-solvable groups to groups with $p^{\prime}$-length one. This latter case naturally leads us to consider a variation of a classical large orbit problem. 
Question 10.1. Suppose that $V$ is a finite faithful completely reducible $F G$ module, where $F$ has characteristic $p$ and $G$ has a normal $p$-complement $K>1$. Let $P \in \operatorname{Syl}_{p}(G)$. Does there exist $v \in \mathbf{C}_{V}(P)$ such that $\left|\mathbf{C}_{K}(v)\right|^{2}<|K|$ ?

Question 10.1 is not trivial, even if $P=1$. In this case, it has an affirmative answer if $K$ is solvable (by [14). Also, Question 10.1 has an affirmative answer if $K$ is nilpotent, and this constitutes the main result of [15]. In some sense, it is unfortunate that our only way to prove that EHZD blocks of $p$-solvable groups are nilpotent is via large orbits. On the other hand, Question 10.1 has interest in its own and it is closely related to the study of $p^{\prime}$-degrees of $p$-solvable groups, so it might deserve some consideration.

Our main result in this section is the following.

Theorem 10.2. Suppose that Question 10.1 has an affirmative answer. If $B$ is an $E H Z D$ block of a p-solvable group $G$, then $B$ is nilpotent.

If $\lambda \in \operatorname{Irr}(N)$ is a character, we write $o(\lambda)$ for its determinantal order. If $N \triangleleft G$, then recall that $\operatorname{Irr}(G \mid \lambda)$ are the irreducible characters of $G$ lying over $\lambda$.

Lemma 10.3. Let $Z \triangleleft G$ and suppose $\lambda \in \operatorname{Irr}(Z)$ is $G$-invariant and that $o(\lambda) \lambda(1)$ is a $p^{\prime}$-number. Assume that $G / Z$ is p-solvable and that $\mathbf{O}_{p^{\prime}}(G / Z)=1$. Suppose that Question 10.1 has an affirmative answer. If there exists an integer $d$ such that $\chi(1)=d$ for all $\chi \in \operatorname{Irr}(G \mid \lambda)$ of $p^{\prime}$-degree, then $G / Z$ is a $p$-group.

Proof. We argue by induction on $|G / Z|$. We may certainly assume that $|G / Z|>1$. By using character triple isomorphisms (see Theorem (3.1) of [32]), we may also assume that $Z$ is a central $p^{\prime}$-group. Thus $Z=\mathbf{O}_{p^{\prime}}(G)$.

Let $U / Z=\mathbf{O}_{p}(G / Z)$ and note that $U>Z$. We suppose that $U<G$, and we seek a contradiction. Let $K / U=\mathbf{O}_{p^{\prime}}(G / U)$. Note that $K>U$. Also, write $U=V \times Z$, and note that $U=\mathbf{O}_{p}(G)$. Also, by Hall-Higman's 1.2.3 Lemma, we have that $\mathbf{C}_{G}(V) \subseteq U \times Z$. If $V_{1}=\Phi(V)$, by elementary group theory we have that $\mathbf{O}_{p^{\prime}}\left(G / Z V_{1}\right)=1$. If $\tilde{\lambda}=1_{V_{1}} \times \lambda \in \operatorname{Irr}\left(V_{1} \times Z\right)$, then $\operatorname{Irr}(G \mid \tilde{\lambda}) \subseteq \operatorname{Irr}(G \mid \lambda)$, and by induction we will conclude that $G / Z V_{1}$ is a $p$-group, and this will prove the theorem. So we may assume that $V=\mathbf{O}_{p}(G)$ is elementary abelian. Hence $\mathbf{C}_{G}(V)=U \times Z$.

Now let $K_{0}=\mathbf{O}^{p}(K)$ and $U_{0}=U \cap K_{0}$. Note that $Z \subseteq U_{0}$ and that $K_{0} / U_{0}=$ $\mathbf{O}_{p^{\prime}}\left(G / U_{0}\right)$. In particular, $\mathbf{O}_{p^{\prime}}\left(G / K_{0}\right)$ is trivial. Also, for all characters $\varphi \in$ $\operatorname{Irr}\left(K_{0} \mid \lambda\right)$, we have that $o(\varphi)$ and $\varphi(1)$ are $p^{\prime}$-numbers (because $K_{0}$ has a normal abelian Sylow $p$-subgroup and $\left.\mathbf{O}^{p}\left(K_{0}\right)=K_{0}\right)$.

Now fix $P \in \operatorname{Syl}_{p}(G)$ and suppose that $\varphi \in \operatorname{Irr}\left(K_{0} \mid \lambda\right)$ is $P$-invariant. Write $T=G_{\varphi}$ for the stabilizer of $\varphi$ in $G$. Hence $|G: T|$ is not divisible by $p$. We claim that $T$ satisfies the hypotheses of the theorem with respect to the character $\varphi$ and the normal subgroup $K_{0} \triangleleft T$. Notice that all $p^{\prime}$-degree members $\psi$ of $\operatorname{Irr}(T \mid \varphi)$ induce to $p^{\prime}$-degree characters of $G$, therefore of degree $d$. Hence $\psi(1)=d / \mid G$ : $T \mid$. Hence to prove the claim, we need to check that $\mathbf{O}_{p^{\prime}}\left(T / K_{0}\right)$ is trivial. Let $W / K_{0}=\mathbf{O}_{p}\left(G / K_{0}\right) \subseteq P K_{0} / K_{0}$, and therefore $W$ stabilizes $\varphi$. Thus $W \subseteq T$ and $\mathbf{O}_{p^{\prime}}\left(T / K_{0}\right)$ centralizes the normal $p$-subgroup $W / K_{0}$. But $\mathbf{O}_{p^{\prime}}\left(G / K_{0}\right)$ is trivial, and Hall-Higman's 1.2.3 Lemma applies to show that $\mathbf{O}_{p^{\prime}}\left(T / K_{0}\right)=1$, as wanted.

By the inductive hypothesis, we conclude that $T / K_{0}$ is a $p$-group; hence $T=$ $K_{0} P$ (since $\left.P \in \operatorname{Syl}_{p}(T)\right)$. We have proved this for all $P$-invariant $\varphi \in \operatorname{Irr}\left(K_{0} \mid \lambda\right)$.

Now let $G_{0}=P K_{0}$. We claim that $G_{0}$ satisfies the hypothesis of the theorem with respect to $Z \triangleleft G_{0}$. Let $\tau \in \operatorname{Irr}_{p^{\prime}}\left(G_{0} \mid \lambda\right)$ and let $\varphi=\tau_{K_{0}} \in \operatorname{Irr}\left(K_{0} \mid \lambda\right)$ which 
is $P$-invariant and irreducible (because $G_{0} / K_{0}$ is a $p$-group). We know that $G_{0}$ is the stabilizer in $G$ of $\varphi$, by the previous paragraphs. Therefore $\tau^{G}=\chi \in \operatorname{Irr}(G)$ is irreducible of $p^{\prime}$-degree. Then $\chi(1)=d$, and we conclude that $\tau(1)=d /\left|G: G_{0}\right|$. So in order to prove the claim we just need to show that $\mathbf{O}_{p^{\prime}}\left(G_{0} / Z\right)=1$. However, we have that $\mathbf{O}_{p^{\prime}}\left(G_{0} / Z\right)$ centralizes $U / Z=\mathbf{O}_{p}(G / Z)$. Since $\mathbf{O}_{p^{\prime}}(G / Z)=1$, we conclude that $\mathbf{O}_{p^{\prime}}\left(G_{0} / Z\right)=1$. If $G_{0}<G$, the inductive hypothesis yields that $G_{0} / Z$ is a $p$-group, which contradicts the fact that $K>U$. Hence we have that $G=P K_{0}$. Thus $\bar{G}=G / U$ has a normal $p$-complement $\bar{K}=K / U$.

Now we have that $\operatorname{Irr}(V)$ is a completely reducible, finite, and faithful $\bar{G}$-module. By using the affirmative answer to Question (10.1), there exists $\beta \in \operatorname{Irr}(V)$ centralized by $P$ such that

$$
\left|K_{\beta} / U\right|^{2}<|K / U|
$$

where $K_{\beta}$ is the stabilizer in $K$ of $\beta$. In other words,

$$
\left|K: K_{\beta}\right|^{2}>|K / U| \text {. }
$$

Now, since $K_{\beta} / V$ is a $p^{\prime}$-group, there exists a unique extension $\hat{\beta} \in \operatorname{Irr}\left(K_{\beta}\right)$ of $\beta$, by using Corollary (8.16) of [23], which has $p$-power order. In particular, this linear character has $Z$ in its kernel, and by uniqueness is $P$-invariant (because $\beta$ is $P$ invariant). Let $\hat{\lambda}=1_{V} \times \lambda \in \operatorname{Irr}(U)$. Since $K_{\beta} / U$ is a $p^{\prime}$-group and $\hat{\lambda}$ is $P$-invariant, then we may find some $\gamma \in \operatorname{Irr}\left(K_{\beta} \mid \hat{\lambda}\right)$ which is $P$-invariant (this is because $\hat{\lambda}^{K_{\beta}}$ has $p^{\prime}$-degree). Now we have that $\gamma \hat{\beta} \in \operatorname{Irr}\left(K_{\beta}\right)$ (because $\hat{\beta}$ is linear) lies over $\beta$. By the Clifford correspondence, we have that $\rho=(\gamma \hat{\beta})^{K} \in \operatorname{Irr}(K)$. This character $\rho$ is $P$-invariant, has $p^{\prime}$-degree $\left|K: K_{\beta}\right| \gamma(1)$ and lies over $\lambda$. Also $\rho_{K_{0}} \in \operatorname{Irr}\left(K_{0}\right)$ is $P$-invariant, has $p^{\prime}$-degree, and therefore it has an extension $\chi \in \operatorname{Irr}(G)$ with $\chi_{K_{0}}=\rho_{K_{0}}$, by using Corollary (8.16) of [23] and the fact that $K_{0}=\mathbf{O}^{p}(G)$. Hence

$$
d=\left|K: K_{\beta}\right| \gamma(1) \geq\left|K: K_{\beta}\right| \text {. }
$$

Therefore,

$$
d^{2} \geq\left|K: K_{\beta}\right|^{2}>|K / U| \text {. }
$$

Now, let $H$ be a $p$-complement of $G$. Hence $H V=K$ and $H \cap V=1$.

Finally, using that $\hat{\lambda}$ is $P$-invariant and $(\hat{\lambda})^{K}$ has $p^{\prime}$-degree, we can find a $P$ invariant $\xi \in \operatorname{Irr}(K \mid \hat{\lambda})$ of $p^{\prime}$-degree. Arguing as before, we have that $\xi$ extends to $G$, and therefore $\xi(1)=d$. However, $\xi_{H} \in \operatorname{Irr}(H \mid \lambda)$. Hence, $d^{2} \leq|H: Z|$ by elementary character theory. However, $|H: Z|=|K: U|$ and this is a contradiction.

(A similar argument gives the same conclusion of Lemma 10.3 if we assume that $\chi^{0} \in \operatorname{IBr}(G)$ for all $\chi \in \operatorname{Irr}(G \mid \lambda)$ of $p^{\prime}$-degree.)

Proof of Theorem 10.2. We argue by induction on $|G|$. Let $Z=\mathbf{O}_{p^{\prime}}(G)$, and let $\lambda \in \operatorname{Irr}(Z)$ be covered by $B$. If $T$ is the stabilizer of $\lambda$ in $G$ and $b$ is the block of $T$ which corresponds to $B$ via Fong-Reynolds ([30, Thm. (9.14)]), then $b$ is an EHZD block. If $T<G$, then $b$ is nilpotent by induction. Thus $B$ is nilpotent by [31. Lemma 1], for instance. Hence, we may assume that $T=G$. In this case, $\operatorname{Irr}(B)=\operatorname{Irr}(G \mid \lambda)$ by $[30$, Thm. (10.20)]. Now we conclude that $G$ has a normal $p$-complement by Lemma 10.3 .

\section{ACKNOWLEDGEMENT}

We would like to thank the reviewer for a careful reading of the manuscript. 


\section{REFERENCES}

[1] J. Alperin, M. Broué, Local methods in block theory, Ann. of Math. (2) 110 (1979), 143-157. MR541333 (80f:20010)

[2] J. An, C. Eaton, Nilpotent blocks of quasisimple groups for odd primes. To appear in J. reine angew. Math., 2010.

[3] J. An, C. Eaton, Nilpotent blocks of quasisimple groups for the prime 2. Preprint, 2009.

[4] C. Bonnafé, Quasi-isolated elements in reductive groups. Comm. Algebra 33 (2005), 23152337. MR2153225 (2006c:20094)

[5] M. Broué, G. Malle, Zyklotomische Heckealgebren. Astérisque 212 (1993), 119-189. MR1235834 (94m:20095)

[6] M. Broué, G. Malle, Generalized Harish-Chandra theory, pp. 85-103 in: Representations of reductive groups. Cambridge University Press, Cambridge, 1998. MR1714151(2000k:20063)

[7] M. Broué, G. Malle, J. Michel, Generic blocks of finite reductive groups. Astérisque 212 (1993), 7-92. MR 1235832 (95d:20072)

[8] M. Broué, J. Michel, Blocs et séries de Lusztig dans un groupe réductif fini. J. Reine Angew. Math. 395 (1989), 56-67. MR983059 (90b:20037)

[9] M. Broué, L. Puig, A Frobenius theorem for blocks. Invent. Math. 56 (1980), 117-128. MR.558864 (81d:20011)

[10] M. Cabanes, M. Enguehard, Representation theory of finite reductive groups. Cambridge University Press, Cambridge, 2004. MR2057756 (2005g:20067)

[11] R. W. Carter, Finite groups of Lie type. Conjugacy classes and complex characters. Wiley Classics Library. John Wiley \& Sons, Chichester, 1993. MR.1266626 (94k:20020)

[12] J.H. Conway, R.T. Curtis, S.P. Norton, R.A. Parker, R.A. Wilson, Atlas of Finite Groups. Clarendon Press, Oxford, 1985. MR827219 (88g:20025)

[13] D. I. Deriziotis, G. O. Michler, Character table and blocks of finite simple triality groups ${ }^{3} D_{4}(q)$. Trans. Amer. Math. Soc. 303 (1987), 39-70. MR896007 (88j:20011)

[14] S. Dolfi, Large orbits in coprime actions of solvable groups, Trans. Amer. Math. Soc. 360, Number 1, (2008), 135-152. MR2341997 (2008i:20027)

[15] S. Dolfi, G. Navarro, Large orbits of elements centralized by a Sylow subgroup. Arch. Math. (Basel) 93 (2009), 299-304. MR2558521 (2010j:20027)

[16] M. Enguehard, Sur les $l$-blocs unipotents des groupes réductifs finis quand $l$ est mauvais. J. Algebra 230 (2000), 334-377. MR.1775796 (2001i:20089)

[17] M. Enguehard, Vers une décomposition de Jordan des blocs des groupes réductifs finis. J. Algebra 319 (2008), 1035-1115. MR2379092 (2008m:20079)

[18] P. Fong, On decomposition numbers of $J_{1}$ and $R(q)$, pp. 415-422 in: Symposia Mathematica, Vol. XIII, Academic Press, London, 1974. MR0357578 (50:10046)

[19] M. Geck, L. Iancu, G. Malle, Weights of Markov traces and generic degrees. Indag. Mathem. 11 (2000), 379-397. MR1813479 (2003b:20009)

[20] G. Hiss, J. Shamash, 3-blocks and 3-modular characters of $G_{2}(q)$. J. Algebra 131 (1990), 371-387. MR:1058552 (91h:20017)

[21] G. Hiss, J. Shamash, 2-blocks and 2-modular characters of the Chevalley groups $G_{2}(q)$. Math. Comp. 59 (1992), 645-672. MR.1134731(93a:20020)

[22] J. E. Humphreys, Defect groups for finite groups of Lie type. Math. Z. 119 (1971), 149-152. MR 0285623 (44:2841)

[23] I. M. Isaacs, Character Theory of Finite Groups. Dover, New York, 1994. MR.1280461

[24] M. Isaacs, S. D. Smith, A note on groups of p-length 1. J. Algebra 38 (1976), 531-535. MR0393215 (52:14025)

[25] G. Lusztig, On the representations of reductive groups with disconnected centre, pp. 157-166 in: Orbites Unipotentes et Représentations, I.Astérisque 168 (1988). MR1021495|(90j:20083)

[26] G. Malle, Die unipotenten Charaktere von ${ }^{2} F_{4}\left(q^{2}\right)$. Comm. Algebra 18 (1990), 2361-2381. MR.1063140 (91k:20015)

[27] G. Malle, Unipotente Grade imprimitiver komplexer Spiegelungsgruppen. J. Algebra 177 (1995), 768-826. MR1358486 (97a:20073)

[28] G. Malle, Almost irreducible tensor squares. Comm. Algebra 27 (1999), 1033-1051. MR 1669100 (99m:20007)

[29] G. Malle, Height 0 characters of finite groups of Lie type. Represent. Theory 11 (2007), 192-220. MR2365640 (2008m:20025) 
[30] G. Navarro, Characters and blocks of finite groups. Cambridge University Press, 1998. MR1632299 (2000a:20018)

[31] G. Navarro, Nilpotent characters. Pacific J. Math. 169 (1995), 343-351. MR 1346259 (96g:20014)

[32] G. Navarro, Brauer characters relative to a normal subgroup. Proc. London Math. Soc. (3) 81 (2000), 55-71. MR 1757046 (2001c:20017)

[33] G. Navarro, G. R. Robinson, Blocks with p-power character degrees. Proc. Amer. Math. Soc. 133 (2005), 2845-2851. MR2159761 (2006d:20020)

[34] H. Pahlings, Normal $p$-complements and irreducible characters. Math. Z. 154 (1977), 243246. MR0439920 (55:12801)

[35] J. B. Olsson, Combinatorics and representations of finite groups. Vorlesungen aus dem Fachbereich Mathematik der Univ. Essen, 20. Universität Essen, Essen, 1993. MR 1264418 (95b:20020)

[36] T. Okuyama, Y. Tsushima, Local properties of $p$-block algebras of finite groups. Osaka J. Math. 20 (1983), 33-41. MR695615 (84i:20012)

[37] W. Reynolds, Blocks and normal subgroups of finite groups. Nagoya Math. J. 22 (1963), 15-32. MR.0153729(27:3690)

FB Mathematik, Technische Universität Kaiserslautern, Postfach 3049, 67653 Kaiserslautern, Germany

E-mail address: malle@mathematik.uni-kl.de

Departament d’Àlgebra, Universitat de València, Dr. Moliner 50, 46100 Burjassot, SPAIN

E-mail address: gabriel.navarro@uv.es 